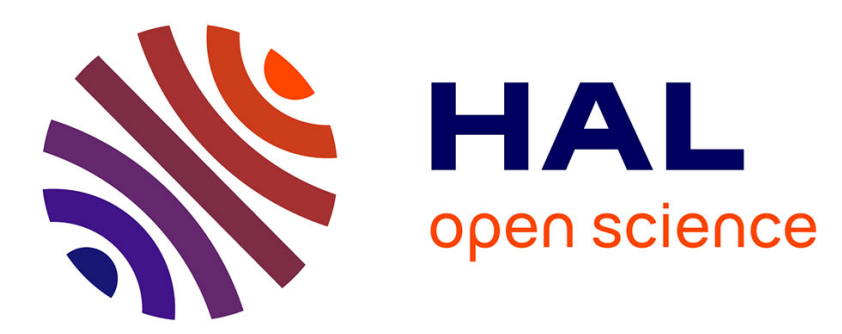

\title{
Les configurations d'aide familiales mobilisées autour des personnes âgées dépendantes en Europe
}

\author{
Roméo Fontaine, Agnès Gramain, Jérôme Wittwer
}

\section{To cite this version:}

Roméo Fontaine, Agnès Gramain, Jérôme Wittwer. Les configurations d'aide familiales mobilisées autour des personnes âgées dépendantes en Europe. Economie et Statistique / Economics and Statistics, 2007, 403-404, pp.97-116. hal-01391611

\section{HAL Id: hal-01391611 \\ https://hal.science/hal-01391611}

Submitted on 3 Nov 2016

HAL is a multi-disciplinary open access archive for the deposit and dissemination of scientific research documents, whether they are published or not. The documents may come from teaching and research institutions in France or abroad, or from public or private research centers.
L'archive ouverte pluridisciplinaire $\mathbf{H A L}$, est destinée au dépôt et à la diffusion de documents scientifiques de niveau recherche, publiés ou non, émanant des établissements d'enseignement et de recherche français ou étrangers, des laboratoires publics ou privés. 


\title{
Les configurations d'aide familiales mobilisées autour des personnes âgées dépendantes en Europe
}

\author{
Roméo Fontaine*, Agnès Gramain** et Jérôme Wittwer*
}

L'enquête Share permet d'étudier l'aide apportée par leurs enfants aux personnes dépendantes âgées d'au moins 65 ans, dans les pays d'Europe. Comment les comportements individuels s'articulent-ils pour donner lieu à des configurations d'aide familiales ? Deux modalités d'implication sont envisagées, selon que les enfants cohabitent ou non avec leur parent dépendant. La proportion de personnes âgées dépendantes recevant un soutien de leur entourage, sous une forme ou sous l'autre, est remarquablement homogène : de $79 \%$ en Suède et aux Pays-Bas à $87 \%$ en Italie. Dans les pays du Nord, la moindre cohabitation inter-générationnelle est en effet compensée par une aide plus fréquente des enfants non cohabitants. Dans les six pays étudiés ici, l'implication des enfants est plus forte lorsque le degré de dépendance du parent est plus élevé et lorsqu'il n'a pas de conjoint.

Cependant, le gradient Nord-Sud se retrouve dans la manière dont les enfants s'adaptent à l'absence de conjoint auprès de leur parent dépendant : accroissement de la cohabitation des filles au Sud, accroissement de l'aide « à distance » des fils comme des filles au Nord. La présence d'un conjoint auprès du parent dépendant semble modifier les logiques d'implication des enfants. Si le parent dépendant bénéficie de l'aide de son conjoint, l'implication des enfants relève de décisions individuelles conduisant à une probabilité de soutien croissante avec le nombre d'enfants. L'implication auprès des parents sans conjoint relèverait au contraire d'une logique de fratrie visant à leur assurer une probabilité de soutien identique quel que soit le nombre de leurs enfants.

Dans les fratries de deux enfants dont le parent est seul, une interaction asymétrique se dessine entre les comportements de l'aîné et du cadet. En moyenne, l'implication du cadet dépendrait de celle de l'aîné (la probabilité de s'impliquer du cadet serait plus faible si l'aîné s'implique lui-même), mais non l'inverse.

* EURISCO-LEGOS (Laboratoire d'économie et de gestion des organisations de santé) - Université Paris-Dauphine ** EURIsCO-LEGOS (Laboratoire d'économie et de gestion des organisations de santé) - Université Paris-Dauphine et Centre d'Études de l'Emploi

Le présent article a été en partie nourri par les réflexions et discussions menées dans le cadre de l'équipe de recherche MEDIPS. Les auteurs tiennent à remercier les différents membres de l'équipe ainsi que les participants du séminaire organisé à l'Irdes pour l'élaboration de ce numéro spécial et les deux relecteurs anonymes pour leurs remarques constructives 
D écrire les modalités d'organisation familiale mises en place autour des personnes âgées dépendantes et analyser leurs déterminants démographiques, économiques et sociaux est important pour nourrir la réflexion sur les politiques sociales en direction des personnes âgées dépendantes. On ne peut orienter, soutenir ou compter sur les solidarités familiales sans s'interroger sur leur sensibilité aux structures familiales, aux trajectoires des individus mais aussi au contexte constitué par le système de protection sociale, l'offre d'aide professionnelle disponible, ou les normes qui pèsent en matière de solidarité familiale. De ce point de vue, la comparaison internationale offre une véritable perspective. C'est en effet le moyen le plus simple de montrer en quoi ces comportements d'aide familiale s'inscrivent dans un contexte social, politique et économique et en quoi, en revanche, ils s'en affranchissent.

Le soutien familial apporté aux personnes âgées dépendantes se trouve depuis une vingtaine d'années au croisement de trois types d'analyse. Une première approche, fortement teintée d'économie de la santé, s'attache à comprendre les modalités de production de soins ou de prise en charge de la dépendance. Elle s'intéresse particulièrement aux questions de substituabilité entre les aides dites formelles et informelles. Sa motivation initiale est d'anticiper l'usage qui pourrait être fait d'une politique de solvabilisation de la demande d'aide formelle, son impact sur la qualité des prises en charge et sur les budgets publics (Ettner, 1994 ; Pezzin et al., 1996 ; Hoeger et al., 1996 ; Sloan et al., 1996). Traitant d'une question connexe, mais avec des enjeux de politique publique différents, la deuxième approche s'interroge sur le comportement d'offre de travail des femmes confrontées à la dépendance de leur parent âgé. Ce sont alors plutôt les politiques de l'emploi qui sont au centre de la réflexion (Boaz et Muller, 1992 ; Pezzin et Schone, 1999). Enfin, une troisième approche met l'accent sur la dynamique de l'aide familiale, cherchant à restituer la genèse des transferts entre ménages apparentés et les processus de désignation des aidants (Hiedemann et Stern, 1999; Engers et Stern, 2002 ; Wolff, 2006 ; Jellal et Wolff, 2002). Il s'agit alors de comprendre comment les structures familiales et leur évolution peuvent affecter le soutien apporté aux personnes âgées dépendantes par leur entourage et l'usage des politiques publiques dans ce domaine (Checkovich et Stern, 2002).

La prise en charge des personnes âgées dépendantes est en effet un moment privilégié pour observer les relations économiques dans la famille. Le terme de "famille» regroupe ici tous les descendants et alliés au premier degré d'une personne (conjoint, enfants, beauxenfants, petits-enfants...) même s'ils ne vivent pas ensemble et se distingue donc du terme de « ménage » qui désigne l'ensemble des personnes partageant un même logement. La prise en charge d'une personne âgée dépendante mobilise en effet son entourage au-delà des seuls membres de son ménage : c'est, pour la France tout au moins, un des rares moments où l'on observe des transferts entre ménages apparentés (Déchaux et Herpin, 2004). On sait cependant encore peu de choses sur le périmètre de la mobilisation familiale et ses déterminants économiques : pourquoi certains membres de la famille sont-ils mobilisés et d'autres non? De même l'existence d'une dimension proprement familiale, collective, des comportements d'aide a été peu explorée par les économistes, dans le contexte français ou plus largement européen (1). Peut-on considérer que les comportements d'implication des différents membres de la famille sont indépendants les uns des autres ou existe-t-il au contraire une logique d'ensemble, une économie familiale de l'aide?

Conduire une telle analyse nécessite des données spécifiques. Il faut en effet disposer d'informations individuelles et contextuelles sur l'ensemble de la famille d'une personne dépendante et non pas seulement sur ceux qui l'aident. L'enquête Share est une des rares enquêtes qui décrive les transferts d'aide entre ménages et fournisse des informations individualisées sur les différents membres de la famille, même s'ils ne cohabitent plus avec la personne dépendante. Ces données portent, qui plus est, sur un échantillon sélectionné dans plusieurs pays dotés de droits de la famille différents (Sosson, 2000). Les comportements d'implication familiale reflétant pour une bonne part les normes morales en matière de solidarité familiale, normes qui ne sont pas sans lien avec celles inscrites dans le droit, l'exploitation de l'enquête Share prend un intérêt supplémentaire. Dans cet article, nous avons retenu six pays, représentant de façon assez schématique différentes positions du curseur entre solidarités collective et familiale dans les principes de leur système de protection sociale: les pays du nord de l'Europe (Suède et Pays-Bas), les pays d'Europe « continentale »

1. Cette remarque concerne les travaux conduits dans une perspective d'analyse économique (les sociologues ont pour leur part largement investi ce champ). II faut cependant mentionner l'exception notable que constituent les travaux de Jellal et Wolff (Jellal et Wolff, 2002) à partir de l'enquête 3G. 
(France et Allemagne) et enfin ceux du sud de l'Europe (Italie et Espagne).

Les configurations d'aide familiales peuvent aussi refléter, d'un pays à l'autre, des différences dans l'offre d'aide professionnelle. La question des liens de substitution ou de complémentarité entre l'aide en nature apportée par les membres de la famille et celle apportée par des professionnels rémunérés constitue un objet à part entière et justifie des investigations spécifiques (Lundborg et al., 2006). Par ailleurs, si le recours aux aides familiales et professionnelles relève d'une même décision, il n'est pas possible d'intégrer l'aide professionnelle dont dispose une personne âgée dépendante comme une information exogène dans l'analyse des comportements de solidarité familiale. Cependant l'implication familiale est prise ici dans une acception très large, sans tenir compte de son intensité. De ce fait, elle apparaît peu sensible aux effets d'offre propres à chaque pays. L'ajustement entre aides familiale et professionnelle toucherait essentiellement à la nature des tâches effectuées par la famille, à la quantité et à l'intensité de l'aide apportée, mais beaucoup moins au fait d'en apporter, sous une forme ou une autre. Les résultats montrent en effet que les comportements d'implication familiale auprès d'un parent âgé sont remarquablement similaires d'un pays à l'autre : l'analyse, par les données de Share, du soutien apporté à leurs parents par les quinquagénaires européens confirme cette forte homogénéité d'un pays à l'autre (Ogg et Renaut, 2006). En revanche, la fréquence de l'aide apportée et les types de tâches effectuées sont beaucoup plus variables. Considérer uniquement le fait de s'impliquer et non l'intensité de l'implication a l'inconvénient de gommer les fortes différences qui peuvent exister entre les membres de l'entourage, mais permet de minimiser les effets d'offre et donc, de centrer l'interprétation sur les logiques proprement familiales.

\section{L'aide aux personnes âgées dépendantes : cohabitation et aide à distance}

D ans le questionnaire Share, l'aide apportée par un membre de l'entourage (famille, voisin, ami, ou autres proches) n'est pas appréciée de la même façon selon que l'individu fait ou non partie du ménage de la personne âgée dépendante (cf. encadré 1). Les personnes exté- rieures au ménage sont interrogées sur l'aide qu'elles apportent aux soins personnels, aux tâches ménagères et administratives. Les membres du ménage ne sont questionnés que sur les soins personnels. En effet, concernant les tâches ménagères et administratives, il est difficile d'isoler ce qui relève d'une aide fournie en réponse à la dépendance de ce qui renvoie à une simple répartition des tâches à l'intérieur du ménage. Pour autant, le fait qu'une personne âgée dépendante ne vive pas seule modifie potentiellement l'implication du reste de la famille, même si les cohabitants ne fournissent aucune aide pour les soins du corps. L'étude du soutien familial apporté aux personnes âgées dépendantes est donc conduite en deux temps. Dans un premier temps, nous considérons la structure des ménages des personnes âgées dépendantes. Tenant compte de cette structure, nous nous intéressons ensuite à l'aide fournie « à distance », c'est-à-dire par des personnes de la famille qui ne cohabitent pas avec la personne aidée. Cette approche change considérablement le rôle d'une variable classiquement retenue parmi les déterminants des transferts entre ménages : celle de la distance entre le ménage de la personne âgée et ceux de ses aidants potentiels. En effet, la cohabitation (correspondant à une distance nulle) devient une modalité même du comportement d'aide, c'est-à-dire du phénomène que l'on cherche à expliquer.

\section{Un peu moins d'un tiers des Européens âgés de plus de 65 ans souffrent d'incapacités}

Il existe différentes approches permettant d'identifier au sein d'une population les personnes ayant des difficultés à réaliser seules certaines activités de la vie quotidienne (Beaufils, 1997). Suivant le degré du besoin d'aide retenu, la proportion de personnes identifiées comme dépendantes peut varier du simple au triple (Colin, 2003).

Nous avons dans ce travail attribué un périmètre très large à la notion de dépendance, afin de disposer d'échantillons suffisamment fournis (cf. encadré 2). Ce choix permet aussi de rendre les échantillons plus comparables d'un pays à l'autre. En effet, l'enquête Share ne porte que sur les personnes vivant en domicile ordinaire : on ne tient donc pas compte de celles qui vivent en établissements d'hébergement collectif. Or les taux d'institutionnalisation des personnes âgées sont très variables d'un pays à l'autre : faibles dans les pays du Sud de l'Europe (2,8\% 
pour l'Espagne et 2,7\% pour l'Italie), ils sont nettement plus importants dans les pays du Nord $(8,2 \%$ pour la Suède et $8,8 \%$ pour les Pays-Bas), les pays " continentaux » ayant une position intermédiaire $(6,8 \%$ pour la France et l'Allemagne) (Assous et Ralle, 2000). On peut craindre que, dans les pays à fort taux d'institutionnalisation, les personnes qui restent à domicile malgré de sévères incapacités, présentent des caractéristiques particulières. Cela pourrait fausser la comparaison avec les pays où l'institutionnalisation est rare, et où reste donc à domicile l'ensemble des personnes lourdement dépendantes. L'impact des comportements d'institutionnalisation sur l'échantillon des personnes demeurant à domicile est donc

Encadré 1

\section{LE SOUTIEN APPORTÉ AUX PERSONNES ÂGÉES DÉPENDANTES DANS L'ENQUÊTE SHARE}

Aide à la personne ou aide au ménage

Un volet du questionnaire Share, intitulé Social Support, permet d'appréhender l'aide reçue de l'entourage par la personne âgée. Cette aide est mesurée différemment selon qu'elle est apportée par une personne extérieure ou par un membre du ménage.

Dans le premier cas, trois types d'aide sont identifiables:

- l'aide aux soins personnels (s'habiller, se laver, manger, entrer ou sortir du lit, aller aux toilettes) ;

- l'aide ménagère (tâches ménagères, courses, déplacement, petits travaux de réparation ou de jardinage) ;

- l'aide aux tâches administratives (remplir des formulaires, régler des questions financières ou juridiques).

Dans le second cas, concernant les aides procurées par les membres du ménage, seules les questions relatives aux soins personnels sont posées. En effet, les deux autres modalités posent traditionnellement des problèmes de déclarations car elles laissent une forte place à l'appréciation des individus sur ce qui peut être identifié, parmi les services que l'on se rend au sein d'un ménage, comme de l'aide liée à la dépendance d'un des membres du ménage et non comme une organisation domestique indépendante de la dépendance.

Par ailleurs, lorsque l'individu vit en couple, les questions relatives à l'aide reçue hors ménage ne permettent pas d'identifier le bénéficiaire de l'aide, la question étant formulée de la façon suivante :

"Lequel/Quel autre de ces membres de votre famille extérieur à votre ménage, de ces amis ou voisins, vous a aidé ... au cours de ces douze derniers mois, vousmême ou votre mari/femme/compagnon/ compagne ? ». L'aide reçue doit donc être comprise au niveau des individus quand ceux-ci vivent sans conjoint, mais au niveau du couple quand l'individu enquêté (le family respondent) vit avec un conjoint.

De l'aide aux personnes dépendantes au soutien apporté par leurs enfants

L'analyse du comportement d'implication des membres de l'entourage des personnes âgées dépendan- tes est focalisée sur les enfants de ces personnes. Cette restriction ne tient pas uniquement à la nature des données disponibles : le même type d'analyse pourrait être mené, à partir des données Share, pour d'autres catégories de personnes apparentées (les frères et sœurs par exemple). Cependant, que l'on prenne un point de vue théorique, en matière d'économie de la famille, ou un point de vue plus opérationnel en matière de politiques sociales, l'intérêt se focalise sur les enfants. Tout d'abord ils représentent la majorité des aidants informels. Ensuite leur comportement n'a rien de systématique, à la différence de celui des conjoints par exemple. Enfin l'encadrement juridique et les normes sociales proposent différents modèles de répartition de la charge entre enfants : égalité des enfants devant l'héritage, équité devant l'obligation alimentaire (pour les pays du Sud), choix d'un successeur (par exemple pour les professions indépendantes), rôle prépondérant des filles, aînesse...

En revanche, la structure du questionnaire Share conduit à restreindre le champ de l'analyse aux fratries comprenant au plus trois enfants. En effet, le questionnaire ne permet d'identifier qu'un maximum de trois aidants (non rémunérés) extérieurs au ménage. Dès lors, pour les familles nombreuses, certains enfants peuvent ne pas être identifiés comme aidant par l'enquête alors qu'ils le sont effectivement. Focaliser l'analyse sur les fratries d'au plus trois enfants permet de limiter ce risque de censure.

\section{Un exemple}

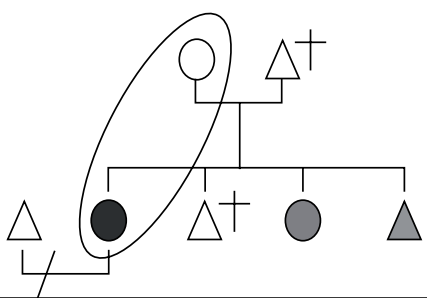

Madame et Monsieur A ont eu quatre enfants, dont trois sont encore en vie. Madame A est veuve et vit avec sa fille aînée, divorcée. Elle déclare recevoir de l'aide pour les tâches administratives de sa deuxième fille et de son fils. On considérera donc que les trois enfants de Madame A sont aidant, la fille aînée (en gris foncé) parce qu'elle cohabite avec elle, ses deux autres enfants (en gris clair) parce qu'ils sont aidants à distance. La famille comporte quatre personnes : Madame A et ses trois enfants alors que le ménage n'en comprend que deux Madame A et sa fille aînée. 
moins fort si l'on considère une population peu dépendante, moins « à risque »d'entrer en maison de retraite.

Trois niveaux d'incapacités sont définis :

- l'incapacité sévère, correspondant aux personnes déclarant avoir des difficultés dans au moins l'une de ces activités de la vie quotidienne : se lever et se mettre au lit, prendre un bain ou une douche, s'habiller ;

- l'incapacité modérée ou légère, correspondant aux personnes déclarant avoir des difficultés dans au moins l'une des activités suivantes : manger, préparer un repas chaud, utiliser les toilettes, se déplacer dans une pièce, aller faire les courses, faire le ménage ou jardiner, prendre des médicaments, passer des appels téléphoniques, gérer de l'argent ou bien utiliser une carte pour se repérer dans un lieu inconnu ;

- l'absence d'incapacité, correspondant aux personnes ne déclarant aucune limitation dans les activités de tous les jours.
Au regard de la définition extrêmement large de la dépendance que représentent les deux premiers degrés, $31 \%$ des Européens (appartenant aux six pays de notre échantillon) âgés de plus de 65 ans souffrent d'incapacités et la moitié d'entre eux sont touchés par au moins une incapacité sévère. Dans les six pays, la proportion de personnes âgées souffrant d'incapacité est fortement croissante avec l'âge. En particulier, plus de quatre Européens âgés de plus de 85 ans sur dix déclarent au moins une incapacité sévère.

\section{Des personnes âgées dépendantes plus isolées au nord de l'Europe}

Dans les six pays considérés, la composition des ménages dans lesquels vivent les individus de plus de 65 ans dépendants présente d'importantes différences. Dans les pays du nord de l'Europe, en particulier la Suède et les Pays-Bas, le nombre moyen de personnes par ménage est inférieur à 1,5 tandis qu'il est, en France et en Allemagne, supérieur à 1,5 et supérieur à 2 dans les pays du sud de l'Europe (Espagne et Italie).

Encadré 2

\section{CONSTRUCTION DE L'ÉCHANTILLON}

Restituer les logiques familiales suppose d'observer la configuration familiale des personnes âgées dépendantes et l'implication de chacun de ses membres. Ceci est possible, à quelques restrictions près, dans l'enquête Share. II faut pour cela constituer un échantillon de personnes âgées dépendantes, dont on observe correctement la configuration familiale.

Articuler les unités statistiques : individus, couples, familles et ménages

L'échantillon Share comprend 22777 individus. Parmi eux, tous n'ont pas répondu à l'intégralité du questionnaire. Le protocole de l'enquête prévoyait en effet de poser certaines questions, telles celles relatives aux enfants de l'individu ou à l'aide non professionnelle reçue, à une seule personne par couple d'enquêtés, le family respondent. En général, chaque ménage ne comprend qu'un family respondent. Parmi les 22777 individus que contient la base, 15930 sont codés family respondent. Parmi eux, 10103 déclarent vivre en couple et 5809 déclarent ne pas avoir de conjoint cohabitant, 18 individus n'ayant pas donné l'information.

Le protocole d'enquête prévoyait aussi d'interviewer les conjoints des individus éligibles. Cependant 3253 conjoints n'ont pas été vus. Le manque d'information sur leur niveau de dépendance est fortement préjudiciable à notre analyse: en effet, lorsque le family respondent est dépendant, son conjoint peut être le principal aidant potentiel ou au contraire une deuxième personne à aider selon qu'il est lui-même dépendant ou non, ce qui modifie sensiblement la donne pour l'implication des enfants dans l'aide. Nous avons dès lors exclu de l'analyse les 3253 individus dont le conjoint n'a pas été vu et les 18 individus pour lesquelles on ne sait pas s'ils cohabitent ou non avec un conjoint. Nous avons de plus exclu de l'analyse les couples comprenant deux individus dépendants: dans ce cas, l'existence d'un cohabitant a en effet une signification très différente puisqu'il ne s'agit pas d'un aidant potentiel.

Parmi les 12660 observations ainsi sélectionnées (5 809 individus vivant sans conjoint et 6851 couples d'individus), 6911 observations, assimilables à des ménages, comprennent un individu âgé de plus de 65 ans. En ne considérant que les données relatives à l'Allemagne, l'Espagne, la France, I'Italie, les Pays-Bas et la Suède, notre sous-échantillon est au final composé de 4655 observations.

L'échantillon étant constitué à partir de taux de sondages variables selon les caractéristiques socio-démo- 
Au-delà de la taille des ménages, c'est leur structure même qui varie d'un pays à l'autre. Ainsi, dans les pays du nord de l'Europe la majorité des individus dépendants vivent seuls (cf. graphique I). Lorsqu'ils cohabitent avec une personne, c'est presque toujours avec leur conjoint. À l'opposé, dans les pays d'Europe du Sud, les personnes âgées dépendantes ne vivent seules que dans environ $35 \%$ des cas. La cohabitation y est donc plus fréquente mais aussi plus large. Les cohabitations avec un conjoint y sont proportionnellement plus faibles que dans les autres pays, mais ceci est plus que compensé par la cohabitation avec un enfant ou avec une autre personne, le plus souvent membre de la famille. La France et l'Allemagne présentent une situation intermédiaire. La part d'individus dépendants vivant seuls y est légèrement plus élevée que dans les pays du sud mais les situations de cohabitation avec un conjoint y sont beaucoup plus fréquentes. Les personnes âgées dépendantes sont donc relativement plus entourées, et donc probablement plus soutenues par des individus cohabitant dans les pays du sud

Encadré 2 (suite)

graphiques des ménages, les statistiques descriptives ont été effectuées sur données pondérées, les poids étant recalculés en fonction des critères de sélection de nos sous-échantillons.

\section{Des échantillons de petite taille}

L'enquête Share n'étant pas centrée sur les personnes dépendantes, les échantillons ainsi constitués sont de petite taille. Les effectifs sont d'autant plus faibles que les échantillons des différents pays ont été le plus souvent traités séparément pour respecter l'hétérogénéité, à travers l'Europe, des dispositifs de production et de financement des soins de long terme ainsi que la probable dispersion des normes de solidarité familiale. Cette remarque concerne avant tout les échantillons construits à l'échelle des personnes âgées dépendantes ou des fratries (cf. tableau A), mais devient moins prégnante dès lors qu'on prend les enfants des personnes dépendantes comme unité statistique (cf. tableau B). Dans ce cas, une personne âgée ayant $n$ enfants compte pour $n$ observations. II nous a semblé important que le lecteur garde la taille des échantillons à l'esprit. C'est pourquoi la taille des échantillons est le plus souvent mentionnée entre parenthèses dans les tableaux, dès que les résultats portent sur des catégories à effectifs réduits, telles que les personnes sans conjoint ou les personnes ayant eu un enfant unique par exemple.

Tableau A

\section{Distribution des ménages selon la présence d'individus dépendants}

\begin{tabular}{|l|c|c|c|c|c|c|c|}
\hline & Suède & Pays-Bas & Allemagne & France & Italie & Espagne & Ensemble \\
\hline $\begin{array}{l}\text { Ménage sans individu } \\
\text { dépendant }\end{array}$ & 572 & 507 & 573 & 357 & 466 & 391 & 2866 \\
$\begin{array}{l}\text { Ménage avec un individu } \\
\text { dépendant }\end{array}$ & 315 & 246 & 274 & 221 & 228 & 324 & 1608 \\
$\begin{array}{l}\text { Ménage avec un couple } \\
\text { d'individus dépendants }\end{array}$ & 20 & 19 & 28 & 22 & 38 & 54 & 181 \\
\hline Ensemble & 907 & 772 & 875 & 600 & 732 & 769 & 4655 \\
\hline
\end{tabular}

Lecture : parmi les 907 ménages suédois présents dans l'échantillon, 572 correspondent à des ménages sans individu dépendant, 315 à des ménages comprenant un seul individu dépendant et 20 à des ménages où les deux membres du couple enquêté sont dépendants.

Champ : ménages comprenant un individu de plus de 65 ans.

Source : enquête Share, 2004.

Tableau B

Distribution des ménages ayant un seul individu dépendant selon le nombre de leurs enfants

\begin{tabular}{|l|c|c|c|c|c|c|c|}
\hline $\begin{array}{c}\text { Nombre } \\
\text { d'enfants }\end{array}$ & Suède & Pays-Bas & Allemagne & France & Italie & Espagne & Ensemble \\
\hline 0 & 14 & 13 & 14 & 19 & 14 & 16 & 15 \\
1 & 17 & 12 & 22 & 19 & 20 & 15 & 17 \\
2 ou 3 & 53 & 51 & 53 & 42 & 46 & 43 & 48 \\
4 ou plus & 16 & 24 & 11 & 20 & 20 & 27 & 20 \\
\hline
\end{tabular}

Lecture : parmi les 315 ménages suédois de l'échantillon comprenant un individu de plus de 65 ans dépendant, 168 correspondent à des ménages dont la personne âgée dépendante a deux ou trois enfants, soit $53 \%$.

Champ : ménages comprenant un seul individu de plus de 65 ans dépendant.

Source : enquête Share, 2004. 
de l'Europe que dans les pays du nord, les pays «continentaux » représentant une situation intermédiaire.

\section{La cohabitation inter-générationnelle comme réponse à la dépendance}

Les personnes qui cohabitent avec la personne âgée dépendante constituent un premier cercle d'aidants potentiels pour pallier les difficultés à réaliser certaines activités de la vie quotidienne. Cependant, la situation est de nature différente selon qu'il s'agit du conjoint ou des enfants : alors que la cohabitation avec un conjoint préexiste la plupart du temps à la survenue d'une incapacité au sein du couple, la cohabitation avec les enfants pourrait constituer une réponse à l'entrée en dépendance du parent. Classiquement on distingue en effet deux modes de cohabitation des enfants avec leurs parents âgés : les corésidences de toujours et les recohabitations, qui impliquent des enfants ayant des caractéristiques bien différentes (AttiasDonfut et Renaut, 1994).

Les personnes de plus de 65 ans cohabitent plus souvent avec un de leurs enfants quand elles souffrent d'incapacité (cf. graphique II). En Espagne en particulier, un quart des ménages de personnes âgées sans incapacité cohabite avec un enfant; cette proportion s'élève à un tiers en cas de dépendance. L'effet du degré de dépendance persiste si l'on raisonne toutes choses égales par ailleurs, en particulier à âge et nombre d'enfants fixés (cf. annexe 1). On constate également d'une part que le degré d'incapacité n'augmente la probabilité de cohabiter avec un enfant que pour les personnes âgées

\section{Graphique I \\ Configuration des ménages des personnes âgées dépendantes}

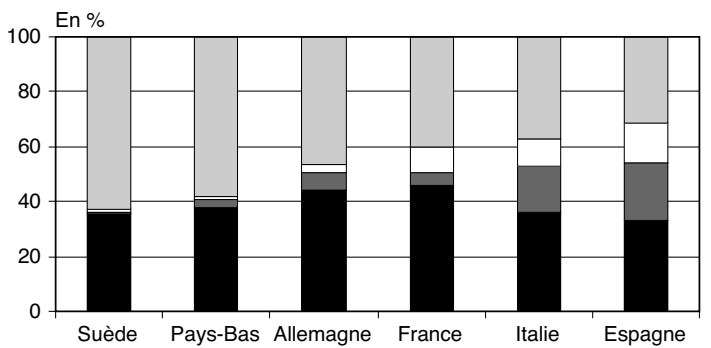

Couple avec ou sans enfant

$\square$ Seul cohabitant avec enfant

$\square$ Seul cohabitant avec autre que enfant $\quad \square$ Seul sans cohabitant

Lecture : un individu " seul » est un individu sans conjoint. Champ : ménages des personnes âgées de plus de 65 ans souffrant d'incapacité sévère ou d'incapacité modérée ou légère, données pondérées.

Source : enquête Share, 2004 sans conjoint et d'autre part que cet effet est d'ampleur similaire dans les trois groupes de pays. Même si la cohabitation inter-générationnelle est d'ampleur variable dans les différents pays étudiés, ces premiers résultats confirment qu'elle constitue, pour une part, dans tous ces pays, une réponse à la dépendance d'un parent âgé, dès lors que celui-ci ne peut compter sur la présence d'un conjoint.

\section{Les enfants, principaux aidants « à distance »}

À l'aide apportée explicitement ou non par les cohabitants s'ajoute potentiellement l'aide fournie « à distance » par des individus qui n'habitent pas avec la personne dépendante. L'expression « aide à distance » s'applique à toutes les personnes extérieures au ménage même si elles habitent le même immeuble ou la même rue que le ménage. Cette aide peut être fournie par des membres de la famille, mais aussi par des voisins, amis, ou autres proches. Le questionnaire Share permet d'identifier l'aide apportée par des personnes extérieures au ménage selon trois modalités : une aide ménagère, une aide administrative et une aide aux soins personnels (cf. encadré 1). Quel que soit le type d'aide, la majorité des aidants «à distance » sont des membres de la famille, entre 70 et $90 \%$, dont les deux tiers sont des enfants de la personne dépendante.

La proportion de ménages recevant une aide « à distance » est différente selon les pays. C'est en Italie et en Espagne, pays où les taux de cohabitation sont les plus forts, que la proportion de ménages aidés «à distance » est la plus faible.

\section{Graphique II \\ Proportion de ménages dans lesquelles une personne âgée cohabite avec un enfant}

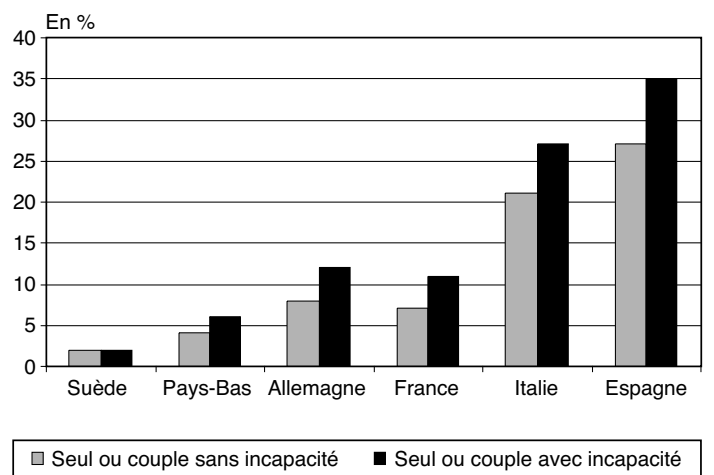

Lecture : un individu " seul » est un individu sans conjoint. Champ : ménages des personnes âgées de plus de 65 ans, données pondérées.

Source : enquête Share, 2004 
En fait, si l'on prend en compte le fait que les personnes dépendantes vivent seules ou non, les six pays retrouvent des profils très similaires. Dans tous les cas, les personnes âgées dépendantes sont proportionnellement plus nombreuses à recevoir une aide « à distance » quand elles vivent seules. En moyenne, $22 \%$ des individus qui vivent avec au moins une autre personne reçoivent une aide «à distance » contre $65 \%$ quand ils vivent seuls. L'Allemagne et l'Espagne se distinguent cependant de leurs voisins européens. En Allemagne, l'aide «à distance » apportée aux individus vivant avec au moins une autre personne est plus élevée que dans les autres pays (37\%), ce qui allège d'autant le poids de la prise en charge qui pèse sur les cohabitants. En Espagne en revanche, l'aide apportée aux personnes dépendantes vivant seules apparaît moins fréquente que dans les autres pays $(51 \%)$. L'aide «à distance » aurait donc un moindre rôle de compensation de l'absence de cohabitants. Par ailleurs, tout comme la cohabitation inter-générationnelle, la fréquence

\section{Graphique III \\ Proportion de ménages recevant une aide à distance de l'entourage selon le niveau d'incapacité}

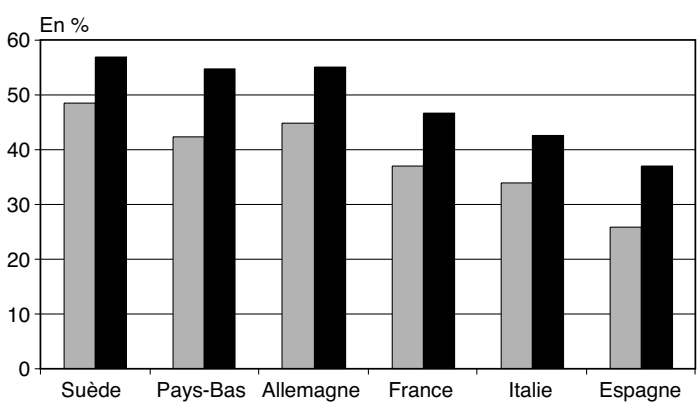

$\square$ Incapacité légère ou modérée - Incapacité sévère

Champ : ménages des personnes âgées de plus de 65 ans souffrant d'incapacité sévère ou d'incapacité modérée ou légère, couples à deux dépendants exclus, données pondérées. Source : enquête Share, 2004 de l'aide «à distance » apportée par l'entourage croît avec le degré d'incapacité (cf. graphique III) : dans chacun des six pays considérés, la proportion de ménages aidés est supérieure de dix points quand le ménage comprend un individu souffrant d'incapacité sévère plutôt que d'incapacité modérée ou légère.

\section{Quatre personnes âgées dépendantes sur cinq sont soutenues par leur entourage}

Si l'on prend en compte les différentes formes d'aide, cohabitation ou aide " à distance », la part de personnes âgées dépendantes soutenue par leur entourage s'avère importante $(83 \%$ en moyenne) et remarquablement similaire dans tous les pays (cf. tableau 1). Plus encore, l'accroissement du soutien familial en réponse à la sévérité de la dépendance est très comparable dans les six pays étudiés (cf. annexe 2).

En revanche, les modalités de ce soutien varient d'un pays à l'autre. Dans les pays du sud de l'Europe (Espagne et Italie), les personnes âgées dépendantes vivent plus rarement seules et cohabitent plus souvent qu'ailleurs avec un de leurs enfants. En contrepartie, elles sont moins nombreuses à recevoir une aide « à distance ». Quand l'aide s'organise à distance, elle est majoritairement hebdomadaire et très souvent quotidienne. Dans les pays du nord de l'Europe (Suède et Pays-Bas), une proportion relativement élevée de personnes âgées dépendantes vivent seules (près de six sur dix), les autres cohabitant essentiellement avec un conjoint. L'aide « à distance » est plus répandue que dans les pays du sud mais elle est dans la plupart des cas occasionnelle. Les pays d'Europe « continentale » (Allemagne et France) présentent une situation intermédiaire entre les deux groupes de pays précédents. Les personnes âgées dépendantes vivent moins souvent seules que dans les pays du nord de l'Europe, mais la cohabitation

Tableau 1

Proportion de personnes âgées dépendantes soutenues par leur entourage

\begin{tabular}{|l|c|c|c|c|c|c|}
\hline & Allemagne & Espagne & France & Italie & Pays-Bas & Suède \\
\hline $\begin{array}{l}\text { Personnes âgées dépendantes cohabitant } \\
\text { avec une autre personne }\end{array}$ & 54 & 68 & 60 & 63 & 42 & 37 \\
$\begin{array}{l}\text { Personnes âgées dépendantes seules } \\
\text { recevant une aide à distance }\end{array}$ & 29 & 16 & 26 & 24 & 37 & 42 \\
$\begin{array}{l}\text { Total des personnes âgées dépendantes } \\
\text { soutenues par l'entourage }\end{array}$ & $\mathbf{8 3}$ & $\mathbf{8 5}$ & $\mathbf{8 6}$ & $\mathbf{8 7}$ & $\mathbf{7 9}$ & $\mathbf{7 9}$ \\
\hline
\end{tabular}

Lecture : en Allemagne, $83 \%$ des personnes âgées dépendantes sont soutenues par leur entourage, sous une forme ou sous une autre ; $54 \%$ cohabitent avec quelqu'un, $29 \%$ vivent seules mais reçoivent une aide à distance.

Champ : ménages des personnes âgées de plus de 65 ans souffrant d'incapacité sévère ou d'incapacité modérée ou légère, couples à deux dépendants exclus, données pondérées.

Source : enquête Share, 2004 
inter-générationnelle est plus rare que dans les pays du sud. La proportion de ménages recevant une aide « à distance » est légèrement plus importante que dans les pays du sud mais moindre que dans les pays du nord. L'aide est moins souvent quotidienne que dans les pays du sud mais les ménages aidés quotidiennement sont deux fois plus nombreux que dans les pays du nord.

\section{Soutenir un parent dépendant : décision individuelle ou décision collective?}

A u-delà de la gravité de leur état, le soutien apporté aux personnes âgées dépendantes pourrait aussi dépendre de la structure familiale. On a vu, par exemple, que la présence d'un conjoint auprès d'une personne dépendante diminue sa probabilité d'être aidée par une personne extérieure au ménage. La taille de la famille, en particulier le nombre des enfants, pourrait aussi entrer en ligne de compte. On pourrait également s'attendre à ce que les personnes âgées reçoivent d'autant plus d'aide de leurs enfants que ceux-ci sont nombreux ; avoir beaucoup d'enfants protégerait contre le risque d'être « abandonné » des siens. On pourrait au contraire envisager que les enfants s'impliquent d'autant moins auprès de leur parent qu'ils ont des frères et sœurs qui peuvent le faire, adoptant ainsi un comportement qualifié de « passager clandestin ».

Analyser le rôle de la structure familiale sur le soutien apporté aux personnes âgées dépendantes suppose d'articuler deux points de vue : celui des personnes dépendantes (quelle est leur probabilité d'être aidée selon qu'elles ont un conjoint, un ou plusieurs enfants ?) et celui de leurs aidants potentiels au premier rang desquels se situent leurs enfants (quelle est leur probabilité d'aider selon que leur parent a un conjoint, selon qu'ils ont ou non des frères et sœurs?). Le soutien reçu par les parents est en effet le résultat de l'agrégation des comportements de leurs différents enfants.

\section{Les enfants pallient l'absence de conjoint auprès de leur parent dépendant}

Si l'on considère comme impliqué auprès de son parent dépendant tout enfant cohabitant ou « aidant à distance », le taux d'implication des enfants s'échelonne de $20 \%$ pour les Pays-Bas à
$38 \%$ pour l'Espagne. L'implication des enfants apparaît très sensible au fait que le parent dépendant puisse ou non compter sur un conjoint. En effet, dans tous les pays le taux d'implication des enfants est nettement plus élevé auprès des parents sans conjoint: on passe par exemple d'un enfant sur dix à quatre enfants sur dix dans le cas de la France (cf. tableau 2).

Pallier l'absence du conjoint se fait cependant de différentes façons selon les pays. De plus, les modalités d'aide semblent peser inégalement sur les fils et les filles (cf. tableaux 3 et 4). Aux Pays-Bas et en Suède, la cohabitation intergénérationnelle apparaît quasi inexistante, même lorsque le parent dépendant est veuf ou divorcé (respectivement $3 \%$ et $1 \%$ des enfants cohabitent avec leur parent dépendant). L'isolement du parent est donc uniquement compensé par une plus large implication des enfants dans l'aide " à distance » et ce dans des proportions similaires pour les fils et les filles. Par exemple, le taux d'implication à distance passe de moins d'un enfant sur dix lorsque le parent peut compter sur un conjoint, à un sur quatre aux PaysBas et à plus d'un sur trois en Suède, quand le parent est isolé. En Allemagne et en France, les enfants pallient aussi l'absence de conjoint auprès de leur parent dépendant par un plus fort taux d'aide à distance (même si les taux de cohabitation inter-générationnelle atteignent presque un enfant sur dix quand le parent est sans conjoint). Mais les taux d'implication des filles et des fils ne sont plus similaires contrairement à ce qui est observé pour les Pays-Bas et la Suède. En Allemagne et en France, lorsqu'un parent est seul, près d'une fille sur deux lui apporte un soutien à distance, contre un fils sur quatre. Enfin, l'Espagne et l'Italie se distinguent

\section{Tableau 2 \\ Taux d'implication des enfants selon la situation matrimoniale de leur parent dépendant}

En \%

\begin{tabular}{|l|c|l|c|}
\hline \multicolumn{4}{|c|}{ Leur parent dépendant vit... } \\
\hline \multicolumn{2}{|c|}{... avec un conjoint } & \multicolumn{2}{c|}{... sans conjoint } \\
\hline Italie & 27 & Espagne & 47 \\
Allemagne & 22 & Allemagne & 43 \\
Espagne & 19 & Italie & 41 \\
France & 11 & France & 41 \\
Pays-Bas & 10 & Suède & 40 \\
Suède & 9 & Pays-Bas & 26 \\
\hline
\end{tabular}

Lecture: en Italie, $27 \%$ des enfants participent au soutien de leur parent dépendant quand ce dernier a un conjoint alors qu'ils sont $41 \%$ quand il n'en a pas.

Champ : enfants de personnes âgées dépendantes de plus de 65 ans ayant un nombre d'enfants inférieur ou égal à trois, couples de dépendants exclus, données pondérées.

Source : enquête Share, 2004 
par le rôle de la cohabitation. Le comportement des fils apparaît insensible à la situation matrimoniale de leur parent dépendant : ils sont, dans tous les cas, moins impliqués qu'ailleurs dans l'aide à distance mais plus souvent cohabitant (aux alentours de $15 \%$ des fils en Espagne et de $25 \%$ en Italie). En revanche, pour les filles, cohabiter avec leur parent semble bien un moyen de compenser l'absence de conjoint. Alors qu'elles ne sont pas plus cohabitantes que les autres Européennes lorsque leurs parents sont encore en couple, environ un tiers d'entre elles vivent avec leur parent dépendant lorsque celuici est seul. Dans ces deux pays, la cohabitation semble donc un support privilégié de l'aide familiale aux personnes âgées dépendantes, avec une signification différente pour les filles et les fils : re-cohabitation de circonstance pour les premières et co-résidence de toujours pour les seconds (Attias-Donfut et Renaut, 1994).

\section{Avoir plus d'enfants n'augmente pas la probabilité d'être aidé pour les personnes âgées dépendantes sans conjoint}

La propension d'un enfant à s'impliquer dans le soutien à un parent dépendant pourrait aussi être influencée par le comportement de ses frères et sœurs (2) et la taille de la fratrie. Le taux d'implication des enfants dont le parent n'a pas de conjoint diminue de façon régulière avec la taille de la fratrie (cf. tableau 5); cette baisse est nette que l'on passe des enfants uniques aux fratries de deux ou des fratries de deux à celles de trois enfants. En revanche, la proportion de parents recevant de l'aide n'évolue pas de façon

2. Nous devons signaler ici que nous n'observons pas l'éventuelle implication des enfants auprès de leurs beaux-parents, nous nous intéressons donc aux interactions au sein de la lignée uniquement.

Tableau 3

Taux d'implication à distance des enfants auprès de leur parent dépendant selon qu'il est seul ou en couple

\begin{tabular}{|l|c|c|c|c|c|c|c|c|c|}
\hline & \multicolumn{3}{|c|}{ Filles } & \multicolumn{3}{c|}{ Fils } & \multicolumn{3}{c|}{ Ensemble des enfants } \\
\cline { 2 - 10 } & $\begin{array}{c}\text { Avec } \\
\text { conjoint }\end{array}$ & $\begin{array}{c}\text { Sans } \\
\text { conjoint }\end{array}$ & $\begin{array}{c}\mathrm{p}^{*} \\
(1)\end{array}$ & $\begin{array}{c}\text { Avec } \\
\text { conjoint }\end{array}$ & $\begin{array}{c}\text { Sans } \\
\text { conjoint }\end{array}$ & $\begin{array}{c}\mathrm{p}^{*} \\
(1)\end{array}$ & $\begin{array}{c}\text { Avec } \\
\text { conjoint }\end{array}$ & $\begin{array}{c}\text { Sans } \\
\text { conjoint }\end{array}$ & $\begin{array}{c}\mathrm{p}^{*} \\
(1)\end{array}$ \\
\hline Allemagne & 21 & 46 & $<0,01$ & 17 & 26 & 0,28 & 19 & 37 & $<0,01$ \\
Espagne & 6 & 44 & $<0,01$ & 10 & 18 & 0,26 & 8 & 29 & $<0,01$ \\
France & 14 & 41 & 0,01 & 2 & 26 & $<0,01$ & 8 & 35 & $<0,01$ \\
Italie & 21 & 19 & 0,88 & 5 & 13 & 0,34 & 14 & 17 & 0,72 \\
Pays-Bas & 6 & 25 & 0,02 & 8 & 23 & 0,04 & 7 & 24 & $<0,01$ \\
Suède & 6 & 46 & $<0,01$ & 10 & 35 & $<0,01$ & 8 & 40 & $<0,01$ \\
\hline
\end{tabular}

Lecture : en Allemagne, $21 \%$ des filles participent au soutien à distance de leur parent dépendant quand ce dernier a un conjoint alors qu'elles sont $46 \%$ quand il n'en a pas. La probabilité de se tromper en disant que ces deux proportions sont différentes est inférieure à une chance sur cent.

Champ : enfants de personnes âgées dépendantes de plus de 65 ans ayant un nombre d'enfants inférieur ou égal à trois, couples de dépendants exclus, données pondérées.

Source : enquête Share, 2004.

Tableau 4

Taux de cohabitation inter-générationnelle des enfants avec leur parent dépendant selon qu'il est seul ou en couple

\begin{tabular}{|l|c|c|c|c|c|c|c|c|c|}
\hline \multirow{2}{*}{} & \multicolumn{3}{|c|}{ Filles } & \multicolumn{3}{c|}{ Fils } & \multicolumn{3}{c|}{ Ensemble des enfants } \\
\cline { 2 - 10 } & $\begin{array}{c}\text { Avec } \\
\text { conjoint }\end{array}$ & $\begin{array}{c}\text { Sans } \\
\text { conjoint }\end{array}$ & $\begin{array}{c}\mathrm{p}^{*} \\
(1)\end{array}$ & $\begin{array}{c}\text { Avec } \\
\text { conjoint }\end{array}$ & $\begin{array}{c}\text { Sans } \\
\text { conjoint }\end{array}$ & $\begin{array}{c}\mathrm{p}^{*} \\
(1)\end{array}$ & $\begin{array}{c}\text { Avec } \\
\text { conjoint }\end{array}$ & $\begin{array}{c}\text { Sans } \\
\text { conjoint }\end{array}$ & $\begin{array}{c}\mathrm{p}^{*} \\
(1)\end{array}$ \\
\hline Allemagne & 2 & 9 & 0,15 & 5 & 9 & 0,37 & 4 & 9 & 0,11 \\
Espagne & 6 & 37 & $<0,01$ & 16 & 14 & 0,78 & 11 & 29 & 0,02 \\
France & 5 & 8 & 0,56 & 1 & 9 & 0,15 & 3 & 9 & 0,17 \\
Italie & 9 & 30 & 0,08 & 22 & 29 & 0,56 & 19 & 26 & 0,10 \\
Pays-Bas & 2 & 2 & 0,80 & 3 & 4 & 0,70 & 3 & 3 & 0,92 \\
Suède & 1 & 1 & 0,82 & 1 & 0 & 0,32 & 1 & 1 & 0,76 \\
\hline \multicolumn{2}{|l|}{ 1. Probabilité critique du test bilatéral d'égalité des taux selon la situation matrimoniale du parent dépendant. } \\
\hline
\end{tabular}

Lecture : en Allemagne, 2 \% des filles cohabitent avec leur parent dépendant quand ce dernier a un conjoint alors qu'elles sont $9 \%$ quand il n'en a pas. La probabilité de se tromper en concluant que ces deux proportions sont différentes est de 15 chances sur cent. Champ : enfants de personnes âgées dépendantes de plus de 65 ans ayant un nombre d'enfants inférieur ou égal à trois, couples de dépendants exclus, données pondérées.

Source : enquête Share, 2004. 
marquée et univoque en fonction de la taille de la fratrie (cf. tableau 6). Dans les six pays étudiés, les évolutions constatées sont variées, peu marquées et statistiquement non significatives. Quel que soit le pays, le fait d'avoir plus d'enfants (au moins jusqu'à trois) n'augmente pas la probabilité d'être aidées pour les personnes âgées dépendantes sans conjoint. Ce résultat est valable même si l'on tient compte explicitement du niveau de dépendance, de l'âge du parent et de la composition de la fratrie (cf. annexe 2) : pour une personne âgée qui n'a pas de conjoint, le nombre de ses enfants vivants n'a pas d'effet significatif sur la probabilité qu'elle reçoive un soutien d'au moins l'un d'entre eux (3). Il semblerait donc que la norme sociale de prise en charge d'un parent dépendant ne pèse pas réellement sur les enfants séparément mais sur les fratries dans leur ensemble. La diminution de la taille moyenne des fratries, constatée dans certains pays européens, devrait donc avoir des conséquences sur les enfants, plus souvent mis à contribution, plutôt que sur les parents dépendants.

Les résultats sont très différents pour les parents dépendants qui vivent encore avec un conjoint puisque la taille de la fratrie augmente la probabilité d'être aidé par leurs enfants (cf. annexe 2). Cette différence dans l'évolution des taux d'implication des enfants en fonction de la taille des fratries, selon que les personnes âgées dépendantes vivent en couple ou non, s'explique très certainement par la nature de l'aide apportée par les enfants. Dans un cas, l'aide des enfants est généralement une aide d'appoint ; dans l'autre cas, il s'agit souvent d'une véritable prise en charge. Lorsque le parent dépendant ne peut compter sur un conjoint, et qu'il s'agit donc pour les enfants de répondre à un besoin d'aide primaire, la «décision de s'impliquer» se prendrait au niveau de la fratrie. L'implication des enfants relèverait au contraire de décisions individuelles lorsque leurs parents sont encore en couple et que l'essentiel de l'aide est déjà assuré par un conjoint. C'est pourquoi, dans ce dernier cas, avoir plus d'enfants augmenterait mécaniquement les chances d'être aidé par au moins l'un d'entre eux.

\section{Dans les fratries de deux enfants : les déterminants de l'implication de l'aîné diffèrent de ceux du cadet}

Considérer le nombre des frères et sœurs n'est cependant pas suffisant pour isoler des logiques de fratries. Ces logiques ne peuvent en effet

3. Nous rappelons que nous ne considérons ici que l'implication des enfants sans tenir compte de l'intensité, de la fréquence ou des types de tâches effectuées.

Tableau 5

Taux d'implication des enfants auprès de leurs parents dépendants sans conjoint, par taille de fratrie

\begin{tabular}{|l|c|c|c|c|c|c|}
\hline & En \% \\
\hline Fratrie de 1 & Allemagne & France & Italie & Espagne & Pays-Bas & Suède \\
Fratrie de 2 & $64(37)$ & $68(24)$ & $76(26)$ & $63(41)$ & $58(10)$ & $53(28)$ \\
Fratrie de 3 & $44(82)$ & $46(58)$ & $40(54)$ & $45(106)$ & $30(66)$ & $44(104)$ \\
\end{tabular}

Lecture : en Allemagne, $64 \%$ des enfants uniques, $44 \%$ des enfants appartenant à une fratrie de deux et $27 \%$ des enfants appartenant à une fratrie de trois s'impliquent dans l'aide à leur parent dépendant. Les effectifs des échantillons apparaissent entre parenthèses. Champ : enfants de personnes âgées dépendantes de plus de 65 ans sans conjoint ayant un, deux ou trois enfants ; données pondérées.

Source : enquête Share, 2004.

Tableau 6

Proportion de personnes âgées dépendantes sans conjoint recevant de l'aide d'un ou de plusieurs enfants

\begin{tabular}{|l|c|c|c|c|c|c|}
\hline & Allemagne & France & Italie & Espagne & Pays-Bas & Suède \\
\hline Fratrie de 1 & $64(37)$ & $68(24)$ & $76(26)$ & $63(24)$ & $58(10)$ & $53(28)$ \\
Fratrie de 2 & $67(41)$ & $63(29)$ & $53(17)$ & $74(53)$ & $44(33)$ & $70(52)$ \\
Fratrie de 3 & $56(21)$ & $61(23)$ & $66(24)$ & $70(28)$ & $41(25)$ & $50(34)$ \\
\hline
\end{tabular}

Lecture : en Allemagne, parmi les personnes dépendantes sans conjoint ayant un enfant unique, $64 \%$ en reçoivent de l'aide. Parmi les personnes ayant deux enfants en vie, 67 \% reçoivent de l'aide d'au moins l'un des deux. Parmi les personnes ayant trois enfants en vie, $56 \%$ reçoivent de l'aide d'au moins l'un des trois. Les effectifs des échantillons apparaissent entre parenthèses.

Champ : personnes âgées dépendantes de plus de 65 ans sans conjoint; données pondérées.

Source : enquête Share, 2004. 
s'éclairer véritablement qu'en considérant les comportements d'implication conditionnels des enfants au sein des fratries, c'est-à-dire en vérifiant dans quelle mesure le comportement d'un enfant constitue une réaction à l'implication de ses frères et sœurs.

Pour mesurer le rôle des interactions dans les décisions d'aide, se concentrer sur les fratries de deux enfants simplifie grandement l'analyse : d'une part on neutralise les effets qui pourraient provenir de la taille des fratries et, d'autre part, l'implication des frères et sœurs se résume à l'implication de l'unique frère ou sœur. On peut alors comparer deux taux d'implication, dits « conditionnels »: le taux d'implication des enfants dont le frère (ou la sœur) est impliqué(e) auprès du parent dépendant, celui des enfants dont le frère (ou la sœur) ne l'est pas (cf. encadré 3). Dans l'ensemble, pour les enfants dont le parent âgé n'a plus de conjoint (4), ces taux d'implication conditionnels sont plutôt plus élevés lorsque l'autre enfant est lui-même aidant. Les résultats sont certes peu systématiques, puisque la différence n'est significative que pour la France et les Pays-Bas et que l'Espagne présente une situation inverse à celle des autres pays (cf. tableau 7). Ils sont cependant contraires aux résultats attendus dans les modèles d'interactions du type "passager clandestin » où chaque enfant souhaite que sa mère (son père) soit aidé(e) par un membre de la fratrie mais préfère que ce soit quelqu'un d'autre que luimême qui s'en charge. Ici, la probabilité d'aider n'est pas plus faible quand on a un frère ou une sœur aidant, ce serait même plutôt l'inverse.

Il convient néanmoins de ne pas interpréter trop rapidement ces résultats en termes d'interactions stratégiques car nous avons implicitement et abusivement supposé que la décision d'un enfant de soutenir son parent ne nous informait sur rien d'autre que sur la décision elle-même. Or, le fait qu'un enfant ne s'implique pas peut révéler que le parent dépendant n'a que peu besoin d'aide, ou, inversement, le fait qu'il s'implique peut informer d'un besoin d'aide important. Plus

4. Les effectifs pour les parents avec conjoint sont trop réduits. D'autre part, d'après les résultats de la section précédente, c'est pour les parents sans conjoint que la question de l'existence d'une logique de fratrie semble se poser.

Tableau 7

Taux d'implication conditionnels des enfants de personnes âgées dépendantes sans conjoint (cas des fratries de deux)

En \%

\begin{tabular}{|l|c|c|c|c|c|c|}
\hline & Allemagne & Espagne & France & Italie & Pays-Bas & Suède \\
\hline Autre enfant non impliqué & $42(48)$ & $53(55)$ & $33(33)$ & $29(34)$ & $21(48)$ & $38(58)$ \\
\hline Autre enfant impliqué & $41(34)$ & $43(51)$ & $56(25)$ & $50(20)$ & $44(18)$ & $52(46)$ \\
\hline
\end{tabular}

Lecture : en Allemagne, 42 \% des individus ayant un frère ou une sœur non impliqué sont eux-mêmes impliqués alors qu'ils sont 41 \% dans ce cas quand ils ont un frère ou une sœur impliqué. Les effectifs des échantillons apparaissent entre parenthèses.

Champ : enfants de personnes âgées dépendantes de plus de 65 ans sans conjoint ayant deux enfants.

Source : enquête Share, 2004.

Encadré 3

PROBABILITÉS CONDITIONNELLES

Cette présentation est inspirée de Solon et al. (1987).

Pour les fratries de 2 enfants, 4 configurations d'aide sont observées : aucun des deux enfants n'aide (soit $N_{00}$ fratries), le premier enfant aide et pas le second (soit $N_{10}$ fratries), le deuxième enfant aide et pas le premier (soit $N_{01}$ fratries), les deux enfants aident (soit $N_{11}$ fratries). Notons $p$ la probabilité inconditionnelle qu'un enfant soit impliqué, $p_{0}$ la probabilité qu'il s'implique sachant que sa sœur (ou son frère) ne s'implique pas et $p_{1}$ la probabilité qu'il s'implique sachant que sa sœur (ou son frère) s'implique. II existe naturellement une relation entre la probabilité inconditionnelle et les probabilités conditionnelles: $p=p_{0} /\left(1+p_{0}-p_{1}\right)$
II est par ailleurs possible d'écrire la probabilité que l'on observe la répartition $\left(N_{00}, N_{10}, N_{01}, N_{11}\right)$ de la manière suivante :

$L=\left[(1-p)\left(1-p_{0}\right)\right]^{N_{00}}\left[(1-p) p_{0}\right]^{N_{01}}\left[\left(1-p_{1}\right) p\right]^{N_{10}}\left[p p_{1}\right]^{N_{11}}$

En remplaçant $p$ par sa valeur, la maximisation de cette vraisemblance conduit aux estimations suivantes :

$\hat{p}_{0}=\left(N_{01}+N_{10}\right) /\left(2 N_{00}+N_{01}+N_{10}\right)$

$\hat{p}_{1}=2 N_{11} /\left(2 N_{11}+N_{01}+N_{10}\right)$

Ce sont ces estimations qui sont présentées dans le tableau 7. 
généralement, les configurations d'aide observées peuvent être fortement influencées par le contexte des prises de décision, c'est-à-dire par les caractéristiques des familles étudiées. Dans ce cas, l'impression de coopération entre les enfants d'une fratrie serait en fait un mimétisme dû au fait que les enfants d'une même fratrie prennent leurs décisions dans un environnement identique, partiellement au moins.

Dans les fratries de deux enfants, estimer deux équations dans un même modèle, expliquant, l'une la probabilité d'implication des aînés, l'autre celle des cadets, permet de tenir compte des effets de contextes familiaux (cf. annexe 3 ). Une fois les effets individuels et les effets de contexte neutralisés, les résultats sont contrastés. Les cadets s'impliquent d'autant moins probablement que leur aîné est lui-même impliqué (pour un seuil de significativité de $10 \%$ ). En revanche, le modèle symétrique qui introduit l'implication du cadet comme variable explicative de l'implication de l'aîné ne révèle pas d'effet significatif (cf. annexe 3). Ceci tendrait à démontrer, d'une part, l'importance des effets de contexte familial, et, d'autre part, l'existence d'interactions asymétriques entre aînés et cadets.

L'asymétrie des comportements entre aînés et cadets ne s'arrête pas aux interactions dans la fratrie. Certains déterminants ne semblent jouer que pour les uns ou les autres. Ainsi, les aînés sont largement influencés par les caractéristiques du parent dépendant, son niveau de dépendance, son âge et son niveau de revenu alors que seul le niveau de revenu influence l'implication des cadets. En revanche, les cadets sont sensibles à la différence d'âge qui les sépare de leur ainé : plus cette différence est importante plus leur probabilité de s'impliquer auprès de leur parent est élevée. On pourrait interpréter ce résultat comme un effet "deuxième enfant unique ». Sensiblement plus jeune que l'aîné, le cadet serait dans une situation objective d'enfant unique dans son enfance qui le conduirait à adopter un comportement d'implication plus proche de celui des enfants uniques. Pour les cadets le genre est également important : les cadettes s'impliquent plus souvent que les cadets surtout quand l'aîné est un garçon.

Concernant la «disponibilité » en temps des frères et sœurs, l'asymétrie entre aînés et cadets demeure mais de manière moins visible. La prise en charge pèse, sans surprise, sur l'enfant, qui, par sa situation matrimoniale ou sa situation visà-vis du marché du travail, est le plus disponible pour aider son parent âgé dépendant. Mais, alors que les cadets s'impliqueraient d'autant moins fréquemment que leur aîné est en couple et sans enfant, l'aîné s'impliquerait d'autant plus fréquemment qu'il est lui-même inactif et que son cadet est actif à plein temps. L'implication des deux enfants est moindre quand ils ont tous les deux un niveau d'éducation élevé ce qui indique sans doute un recours plus important à une aide professionnelle.

De manière générale, dans l'estimation proposée les variables jouent plutôt de manière relative : l'effet du statut d'un membre de la fratrie est fonction du statut du frère ou de la sœur. Le choix de variables croisées (âge, genre, statut vis-à-vis de l'activité, diplôme) a été guidé par les estimations qui ont montré qu'elles apparaissaient sensiblement plus significatives sous cette forme.

Les effets de contexte semblent ainsi particulièrement subtils et étroitement dépendants de la composition de la fratrie ; il serait cependant nécessaire d'aller plus loin dans la modélisation pour s'assurer qu'ils ne cachent pas des effets d'interactions entre les frères et sœurs. Néanmoins, cela nous conduirait trop loin au regard de l'ambition de cet article (5). En dernier lieu, on notera que l'on ne peut mettre en évidence d'effet pays si ce n'est pour les aînés en Suède (pour un seuil de significativité de $10 \%$ ). Le croisement d'indicatrice pays avec les autres variables indépendantes du modèle a été testé sans mettre en évidence d'effet significatif.

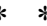

Des différents éclairages portés ici sur l'aide aux personnes âgées dépendantes à travers l'Europe, nous pouvons retenir deux principaux enseignements concernant le fonctionnement de la famille en tant que groupe de solidarité ou de production.

Tout d'abord, il semble difficile d'isoler les comportements d'aide de la structure familiale dans laquelle ils s'inscrivent. Plusieurs résultats en témoignent. Le fait qu'une personne âgée puisse compter sur son conjoint change sensiblement le comportement d'implication

5. À notre connaissance, très peu de travaux empiriques se sont attelés à ce type de modélisation dans le contexte de la prise en charge d'un parent dépendant; on pourra néanmoins se référer à Hiedemann et Stern (1999), pour un exemple de modélisation structurelle. 
de ses enfants. L'isolement du parent semble associé à l'existence de comportements collectifs chez les enfants, repérables par des régularités à l'échelle des fratries plus qu'à celle des enfants. Enfin, effet de contexte ou d'interaction, il semble bien que la place occupée dans la fratrie modifie non pas tant la probabilité de s'impliquer, que le comportement d'implication lui-même. Les aînés sont plus influencés par les caractéristiques de leur parent dépendant, tandis les cadets le sont davantage par celles de leurs aînés. Pour autant, et c'est un enseignement plus inattendu que le premier, les normes de solidarité familiale à l'égard des ascendants semblent très homogènes à travers l'Europe, pour le moins au niveau de généralité que nous avons adopté. Ainsi, la proportion de parents ne recevant aucun soutien ou bien le taux d'implication des enfants auprès de leurs parents sans conjoint sont très voisins d'un pays à l'autre. De même l'adaptation de l'implication familiale au degré de dépendance de la personne âgée apparaît similaire. Au-delà de ces similitudes, réapparaissent bien sûr des modalités d'implication (cohabitation ou aide « à distance ») ou de répartition (entre filles et fils) propres à chaque pays. Mais on ne peut qu'être frappé par la conformité des comportements familiaux de soutien, en particulier aux parents qui ne peuvent plus compter sur la présence d'un conjoint valide. Cela suggère l'existence d'une norme morale de solidarité assez identique selon les pays qui pèserait sur les aidants potentiels de première ligne.

Pour la réalisation de cette recherche, nous nous sommes servis des données issues de la première version (Release 1) de la vague 1 de l'enquête Share réalisée en 2004. Celle-ci est préliminaire et pourrait contenir des erreurs qui seront corrigées dans des versions ultérieures. La collecte des données de Share a été principalement financée par le $5^{e}$ Programme Cadre de la Communauté Européenne (Projet QLK6-CT-2001-00360 sur le thème de la qualité de la vie). Des fonds proviennent également du US National Institute on Aging (U01 AG09740-13S2, P01 AG005842, P01 AG08291, P30 AG12815, Y1-AG-4553-01 et OGHA 04-064). La collecte des données a été financée par des institutions nationales en Autriche (Austrian Science Foundation, FWF), en Belgique (Politique Scientifique Fédérale) et en Suisse (BBW/OFES/UFES). Pour la France, elle a bénéficié de financements complémentaires apportés par la Cnav, la Cnam, le Cor, la Drees, la Dares, la Caisse des Dépôts et Consignations et le Commissariat Général du Plan. Les données et la méthodologie de l'enquête sont respectivement présentées dans Börsch-Supan et al. (2005) et Börsch-Supan et Jürges (2005).

\section{BIBLIOGRAPHIE}

Assous L. et Ralle P. (2000), « La prise en charge de la dépendance des personnes âgées : une mise en perspective internationale », Conférence internationale de recherche en sécurité sociale «an $2000 »$, Helsinki, 25-27 septembre.

Attias-Donfut C. et Renaut S. (1994), «Vieillir avec ses enfants. Corésidence de toujours et recohabitation », Les cahiers de sociologie de la famille, $\mathrm{n}^{\mathrm{o}} 1$, pp. 57-80.

Beaufils B. (1997), « De la mesure de la dépendance ", Actualité et dossier en santé publique, $\mathrm{n}^{\circ} 20, \mathrm{pp}$. VI-VII.

Béliard A., Fontaine R., Perrin-Heredia A., Soutrenon E. et Weber F. (2006), «Care and
Tranmission. The Case of French Alzheimer's Diseased», Law and Society Association, Baltimore, Workshop «Care, Law and Public Policy », 8 juillet.

Boaz R. F. et Muller C. F. (1992), « Paid Work and Unpaid Help by Caregivers of the Disabled and Frail Elders ", Medical Care, vol. 30, n $^{\circ} 2$, pp. 149-158.

Bonsang A. (2007), «How do Middle-Aged Children Allocate Time and Money Transfers to Their Older Parents in Europe ? », Empirica, vol. $34, \mathrm{n}^{\circ} 2$, pp. 171-188.

Börsch-Supan A., Brugiavini A, Jürges H., Mackenbach J., Siegrist J. et Weber G. (2005), 
« Health, Ageing and Retirement in Europe - First Results from the Survey of Health, Ageing and Retirement in Europe », Mannheim : MEA.

Checkovich T. et Stern S. (2002), « Shared Caregiving Responsibilities of Adult Siblings with Elderly Parents », Journal of Human Resources, vol. $37, \mathrm{n}^{\circ} 3$, pp. 441-478.

Colin C. (2003), «Que nous apprend 1'enquête HID sur les personnes âgées dépendantes, aujourd'hui et demain? », in « Handicaps, incapacités, dépendance », Revue française des affaires sociales, $\mathrm{n}^{\circ} 1-2$, janvier-juin, pp. 77-101.

Déchaux J.-H. et Herpin N. (2004), « Entraide familiale, indépendance économique et sociabilité », Économie et Statistique, n 373, pp. 3-32.

Engers M. et Stern S. (2002), « Long-Term Care and Family Bargaining », International Economic Review,vol. 43, n 1, pp. 73-114.

Ettner S.L. (1994), "The Effect of Medicaid Home Care Benefit on Long-Term Care Choices of the Elderly », Economic Inquiry, vol. 32, n 1 , pp. 103-127.

Fontaine R., Gramain A. et Wittwer J. (2006), «Caring for Elderly Suffering from Mental and Behavioural Disorders: Contributions and Coordination of Informal Caregivers », ECHE 2006, Budapest, 6-9 juillet.

Hiedemann B. et Stern S. (1999), «Strategic Play Among Family Members When Making Long-Term Care Decisions",, Journal of Economic Behavior and Organization, vol. 40, $\mathrm{n}^{\mathrm{o}} 1$, pp. 29-57.

Hoeger T.J., Picone G.A. et Sloan F.A. (1996), «Public Subsidies, Private Provision of Care and Living Arrangements of the Elderly », The Review of Economics and Statistics, vol. 78, $\mathrm{n}^{\circ} 3$, pp. 428-440.

Jellal M. et Wolff F.-C. (2002), «Aides aux parents âgés et allocation intra-familiale », Revue économique, vol. 53, $\mathrm{n}^{\circ}$ 4, pp. 561-583.

Lundborg P, Lindgren B. et Bolin K. (2006), « Informal and Formal Care among Single-Living Elderly in Europe », ECHE 2006, Budapest, 6-9 juillet.
Maddala G. S. (1983), Limited-dependent and qualitative variables in econometrics, coll. Econometric Society Monographs, Ed.Cambridge University Press (Cambridge), 401 pages.

Ogg J. et Renaut S. (2006), « Les quinquagénaires européens et leurs parents. De la famille ou de l'État, qui doit s'occuper des ascendants?», Informations sociales, $\mathrm{n}^{\circ} 134$, pp. 28-39.

Pezzin L. E., Kemper P. et Reschovsky J. D. (1996), «Does Publicly Provided Home Care Substitute for Family Care ?ExperimentalEvidence With Endogenous Living Arrangements », Journal of Human Resources, vol.31, n 3, pp. 650-676.

Pezzin L. E. et Schone B. S. (1999), « Intergenerational Household Formation Labor Supply, and Informal Caregiving: A Bargaining Approach », Journal of Human Resources, vol. 34, $\mathrm{n}^{\mathrm{o}} 3$, pp. 475-503.

Sloan F. A., Hoeger T. J. et Picone G. (1996), «Effects of Strategic Behaviour and Public Subsidies on Families'Saving and Long-Term Care Decision », in Long-Term Care : Economic Issues and Policy Solution, ouvrage collectif sous la direction de Eisen R. et Sloan F., coll. Developments in Health Economics and Public Policy, Ed. Kluwer academic publishers (Dordrecht), pp. 45-78.

Solon G., Corcoran M., Gordon R. et Laren D. (1988), « Sibling and IntergenerationalCorrelations in Welfare Program Participation », Journal of Human Resources, vol. 23, n 3, pp. 288-396.

Sosson J. (2000), «Analyse comparée des liens juridiques fondant la solidarité alimentaire légale dans les pays occidentaux », Obligation alimentaire et solidarités familiales, ouvrage collectif sous la direction de Choquet L.-H. et Sayn I., coll. Droit et Société, vol. 31, ch. 3.

Stern S. (1995), « Estimating Family Long-Term Care Decisions in the Presence of Endogeneous Child Characteristics », Journal of Human Resources, vol. 30, n 3, pp. 551-580.

Wolff F.-C. (2000), «Transferts et redistribution familiale collective ", Revue économique, vol. 51, $\mathrm{n}^{\circ}$ hors-série, pp. 143-162.

Wolff F.-C. (2006), « Les transferts ascendants au Bangladesh : une décision familiale », L'Actualité Economique, vol. 82, n 1-2. 


\section{DÉTERMINANTS DE LA PROBABILITÉ DE COHABITATION AVEC UN ENFANT (RÉGRESSION LOGISTIQUE)}

\begin{tabular}{|c|c|c|c|c|}
\hline & \multicolumn{2}{|c|}{ Personnes âgées sans conjoint } & \multicolumn{2}{|c|}{ Personnes âgées avec conjoint } \\
\hline & Coefficient & Écart-type & Coefficient & Écart-type \\
\hline Constante & $-1,79^{\star \star \star}$ & 0,11 & $-2,20^{\star \star *}$ & 0,16 \\
\hline $\begin{array}{l}\text { Indicatrice pays } \\
\text { Suède } \\
\text { Hollande } \\
\text { Allemagne } \\
\text { France } \\
\text { Italie } \\
\text { Espagne }\end{array}$ & $\begin{array}{c}-2,14^{\star \star \star} \\
-0,84^{\star \star \star} \\
\text { n.s. } \\
\text { Réf. } \\
1,49^{\star \star \star} \\
1,59^{\star \star \star}\end{array}$ & $\begin{array}{c}0,33 \\
0,22 \\
\text { n.s. } \\
\text { Réf. } \\
0,14 \\
0,13\end{array}$ & $\begin{array}{c}-1,27^{\star \star \star} \\
-0,75^{\star \star \star} \\
-0,36^{\star \star} \\
\text { Réf. } \\
1,44^{\star \star \star} \\
1,46^{\star \star \star}\end{array}$ & $\begin{array}{c}0,24 \\
0,21 \\
0,18 \\
\text { Réf. } \\
0,13 \\
0,14\end{array}$ \\
\hline $\begin{array}{l}\text { Âge de la personne âgée } \\
\text { ou âge moyen du couple } \\
\text { De } 65 \text { à } 74 \text { ans } \\
\text { De } 75 \text { à } 84 \text { ans } \\
\text { Plus de } 85 \text { ans }\end{array}$ & $\begin{array}{c}\text { Réf. } \\
-0,26^{\star \star \star} \\
0,32^{\star \star \star}\end{array}$ & $\begin{array}{c}\text { Réf. } \\
0,09 \\
0,11\end{array}$ & $\begin{array}{c}\text { Réf. } \\
-0,70^{\star \star \star} \\
\text { n.s. }\end{array}$ & $\begin{array}{c}\text { Réf. } \\
0,19 \\
0,40\end{array}$ \\
\hline $\begin{array}{l}\text { Niveau d'incapacité } \\
\text { Sans incapacité } \\
\text { Avec incapacité }\end{array}$ & $\begin{array}{c}\text { Réf. } \\
0,30^{\star \star \star}\end{array}$ & $\begin{array}{c}\text { Réf. } \\
0,10\end{array}$ & $\begin{array}{l}\text { Réf. } \\
0,07\end{array}$ & $\begin{array}{l}\text { Réf. } \\
0,09\end{array}$ \\
\hline $\begin{array}{l}\text { Variables croisées } \\
\text { Incapacité et Pays } \\
\text { Avec incapacité et Suède } \\
\text { Avec incapacité et Hollande } \\
\text { Avec incapacité et Allemagne } \\
\text { Avec incapacité et France } \\
\text { Avec incapacité et Italie } \\
\text { Avec incapacité et Espagne }\end{array}$ & $\begin{array}{l}\text { n.s. } \\
\text { n.s. } \\
\text { n.s. } \\
\text { Réf. } \\
\text { n.s. } \\
\text { n.s. }\end{array}$ & $\begin{array}{l}\text { n.s. } \\
\text { n.s. } \\
\text { n.s. } \\
\text { Réf. } \\
\text { n.s. } \\
\text { n.s. }\end{array}$ & $\begin{array}{l}\text { n.s. } \\
\text { n.s. } \\
\text { n.s. } \\
\text { Réf. } \\
\text { n.s. } \\
\text { n.s. }\end{array}$ & $\begin{array}{l}\text { n.s. } \\
\text { n.s. } \\
\text { n.s. } \\
\text { Réf. } \\
\text { n.s. } \\
\text { n.s. }\end{array}$ \\
\hline $\begin{array}{l}\text { Nombre d'enfants } \\
\text { Un enfant } \\
\text { Plusieurs enfants }\end{array}$ & $\begin{array}{l}\text { Réf. } \\
0,14^{*}\end{array}$ & $\begin{array}{c}\text { Réf. } \\
0,08\end{array}$ & $\begin{array}{l}\text { Réf. } \\
\text { n.s. }\end{array}$ & $\begin{array}{l}\text { Réf. } \\
\text { n.s. }\end{array}$ \\
\hline Nombre d'observations & 1758 & & 2233 & \\
\hline
\end{tabular}

Lecture : la variable expliquée vaut 1 lorsque la personne dépendante (et son conjoint le cas échéant) cohabite avec un de leurs enfants, 0 sinon; un coefficient positif signifie que la modalité est associée à une plus forte probabilité de cohabiter. La valeur du coefficient correspond au logarithme de l'odds-ratio; ${ }^{* * *}$ significatif à $1 \%$, ${ }^{* *}$ significatif à $5 \%$, * significatif à $10 \%$.

Champ : ménage comprenant un individu de plus de 65 ans ayant au moins un enfant.

Source : enquête Share, 2004. 
DÉTERMINANTS DE LA PROBABILITÉ D'ÊTRE SOUTENU PAR AU MOINS UN DE SES ENFANTS (RÉGRESSION LOGISTIQUE)

\begin{tabular}{|c|c|c|c|c|}
\hline & \multirow{2}{*}{\multicolumn{2}{|c|}{$\begin{array}{c}\text { Modèle } 1 \\
\begin{array}{c}\text { Personnes âgées dépendantes } \\
\text { avec conjoint }\end{array}\end{array}$}} & \multirow{2}{*}{\multicolumn{2}{|c|}{$\begin{array}{c}\text { Modèle } 2 \\
\begin{array}{c}\text { Personnes âgées dépendantes } \\
\text { sans conjoint }\end{array}\end{array}$}} \\
\hline & & & & \\
\hline & coefficient & Écart-type & coefficient & Écart-type \\
\hline Constante & $-1,16^{\star \star \star}$ & 0,25 & 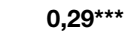 & 0,11 \\
\hline $\begin{array}{l}\text { Indicatrice pays } \\
\text { Allemagne } \\
\text { France } \\
\text { Pays-Bas } \\
\text { Suède } \\
\text { Italie } \\
\text { Espagne }\end{array}$ & 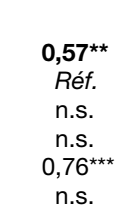 & $\begin{array}{c}0,24 \\
\text { Réf. } \\
\text { n.s. } \\
\text { n.s. } \\
0,27 \\
\text { n.s. }\end{array}$ & $\begin{array}{c}\text { n.s. } \\
\text { Réf. } \\
-0,69^{\star *} \\
\text { n.s. } \\
\text { n.s. } \\
\text { n.s. }\end{array}$ & $\begin{array}{c}\text { n.s. } \\
\text { Réf. } \\
0,27 \\
\text { n.s. } \\
\text { n.s. } \\
\text { n.s. }\end{array}$ \\
\hline $\begin{array}{l}\text { Niveau d'incapacité de la } \\
\text { Léger ou modéré } \\
\text { Sévère }\end{array}$ & $\begin{array}{r}\text { endante } \\
\text { Réf. } \\
\mathbf{0 , 3 3}^{\star *}\end{array}$ & $\begin{array}{c}\text { Réf. } \\
0,12\end{array}$ & $\begin{array}{c}\text { Réf. } \\
0,19^{\star *}\end{array}$ & $\begin{array}{c}\text { Réf. } \\
0,10\end{array}$ \\
\hline $\begin{array}{l}\text { Âge de la personne âgée c } \\
\text { De } 65 \text { à } 74 \text { ans } \\
\text { De } 75 \text { à } 84 \text { ans } \\
\text { Plus de } 85 \text { ans }\end{array}$ & $\begin{array}{l}\text { Réf. } \\
\text { n.s. } \\
\text { n.s. }\end{array}$ & $\begin{array}{l}\text { Réf. } \\
\text { n.s. } \\
\text { n.s. }\end{array}$ & $\begin{array}{l}\text { Réf. } \\
\text { n.s. } \\
\mathbf{0 , 4 9 * \star \star}\end{array}$ & $\begin{array}{l}\text { Réf. } \\
\text { n.s. } \\
0,15\end{array}$ \\
\hline $\begin{array}{l}\text { Nombre d'enfants dans la } \\
\text { Un } \\
\text { Deux } \\
\text { Trois }\end{array}$ & $\begin{array}{c}-0,57^{\star \star \star} \\
\text { Réf. } \\
0,48^{\star \star \star}\end{array}$ & $\begin{array}{c}0,21 \\
\text { Réf. } \\
0,17\end{array}$ & $\begin{array}{l}\text { n.s. } \\
\text { Réf. } \\
\text { n.s. }\end{array}$ & $\begin{array}{l}\text { n.s. } \\
\text { Réf. } \\
\text { n.s. }\end{array}$ \\
\hline $\begin{array}{l}\text { Présence de filles dans la } \\
\text { Aucune } \\
\text { Au moins une } \\
\text { Au moins une et Allemagne } \\
\text { Au moins une et Pays- Bas } \\
\text { Au moins une et Suède } \\
\text { Au moins une et Italie } \\
\text { Au moins une et Espagne }\end{array}$ & $\begin{array}{l}\text { indicatrice } \\
\text { Réf. } \\
\text { n.s. } \\
\text { n.s. } \\
\text { n.s. } \\
\text { n.s. } \\
\text { n.s. } \\
\text { n.s. }\end{array}$ & $\begin{array}{l}\text { Réf. } \\
\text { n.s. } \\
\text { n.s. } \\
\text { n.s. } \\
\text { n.s. } \\
\text { n.s. } \\
\text { n.s. }\end{array}$ & 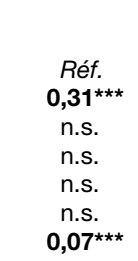 & $\begin{array}{l}\text { Réf. } \\
0,11 \\
\text { n.s. } \\
\text { n.s. } \\
\text { n.s. } \\
\text { n.s. } \\
0,22\end{array}$ \\
\hline Nombre d'observations & 493 & & 557 & \\
\hline
\end{tabular}

Lecture : la variable expliquée vaut 1 lorsque la personne reçoit du soutien d'au moins l'un de ses enfants, sous une forme ou sous une autre (cohabitation ou aide à distance), et vaut 0 quand la personne ne reçoit aucun soutien d'aucune sorte d'aucun de ses enfants; un coefficient positif signifie que la modalité est associée à une plus forte probabilité de recevoir du soutien; la valeur du coefficient correspond au logarithme de l'odds-ratio ; *** significatif à $1 \%$, ** significatif à $5 \%$, * significatif à $10 \%$.

Champ : ménage comprenant un individu dépendant de plus de 65 ans ayant un, deux ou trois enfants.

Source : enquête Share, 2004. 
ANNEXE 3

L'IMPLICATION DES CADETS ET AÎNÉS DE FRATRIES DE DEUX ENFANTS AUPRES D'UN PARENT DEPENDANT SANS CONJOINT (RÉGRESSION DE TYPE PROBIT BI-VARIÉ)

\begin{tabular}{|c|c|c|c|c|}
\hline & \multicolumn{2}{|c|}{$\begin{array}{l}\text { Implication du cadet } \\
\text { Équation } n^{\circ} 1\end{array}$} & \multicolumn{2}{|c|}{$\begin{array}{l}\text { Implication de l'aîné } \\
\text { Équation } n^{\circ} 2\end{array}$} \\
\hline & Coefficient & Écart-type & Coefficient & Écart-type \\
\hline Constante & $-2,96^{\star \star}$ & 1,37 & 1,05 & 1,15 \\
\hline $\begin{array}{l}\text { Indicatrice pays } \\
\text { Allemagne } \\
\text { France } \\
\text { Pays-Bas } \\
\text { Suède } \\
\text { Italie } \\
\text { Espagne }\end{array}$ & $\begin{array}{l}\text { n.s. } \\
\text { Réf. } \\
\text { n.s. } \\
\text { n.s. } \\
\text { n.s. } \\
\text { n.s. }\end{array}$ & $\begin{array}{l}\text { n.s. } \\
\text { Réf. } \\
\text { n.s. } \\
\text { n.s. } \\
\text { n.s. } \\
\text { n.s. }\end{array}$ & 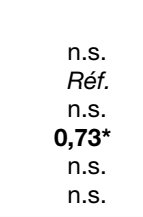 & $\begin{array}{l}\text { n.s. } \\
\text { Réf. } \\
\text { n.s. } \\
0,37 \\
\text { n.s. } \\
\text { n.s. }\end{array}$ \\
\hline $\begin{array}{l}\text { Caractéristiques de la personne â } \\
\text { Niveau d'incapacité } \\
\text { Léger ou modéré } \\
\text { Sévère }\end{array}$ & $\begin{array}{l}\text { ndante } \\
\qquad \begin{array}{l}\text { Réf. } \\
\text { n.s. }\end{array}\end{array}$ & $\begin{array}{l}\text { Réf. } \\
\text { n.s. }\end{array}$ & $\begin{array}{l}\text { Réf. } \\
\mathbf{0 , 5 4 ^ { \star \star }}\end{array}$ & $\begin{array}{l}\text { Réf. } \\
0,22\end{array}$ \\
\hline $\begin{array}{l}\text { Âge de la personne âgée dépend } \\
\text { De } 65 \text { à } 74 \text { ans } \\
\text { De } 75 \text { à } 84 \text { ans } \\
\text { Plus de } 85 \text { ans }\end{array}$ & $\begin{array}{l}\text { Réf. } \\
\text { n.s. } \\
\text { n.s. }\end{array}$ & $\begin{array}{l}\text { Réf. } \\
\text { n.s. } \\
\text { n.s. }\end{array}$ & $\begin{array}{l}\text { Réf. } \\
1,01^{\star \star \star} \\
1,51^{\star \star *}\end{array}$ & $\begin{array}{l}\text { Réf. } \\
0,32 \\
0,39\end{array}$ \\
\hline $\begin{array}{l}\text { Revenu de la personne âgée dépe } \\
\text { Premier quartile } \\
\text { Deuxième quartile } \\
\text { Troisième quartile } \\
\text { Quatrième quartile }\end{array}$ & $\begin{array}{l}\text { lans son pays } \\
-0,57^{\star \star} \\
\text { Réf. } \\
\text { n.s. } \\
\text { n.s. }\end{array}$ & $\begin{array}{l}\text { tenance } \\
0,27 \\
\text { Réf. } \\
\text { n.s. } \\
\text { n.s. }\end{array}$ & $\begin{array}{l}-0,59^{\star \star} \\
\text { Réf. } \\
-0,62^{\star \star} \\
\text { n.s. }\end{array}$ & $\begin{array}{l}0,298 \\
\text { Réf. } \\
0,27 \\
\text { n.s. }\end{array}$ \\
\hline $\begin{array}{l}\text { Caractéristiques du cadet } \\
\text { Âge }\end{array}$ & $0,04^{\star}$ & 0,02 & & \\
\hline $\begin{array}{l}\text { Situation matrimoniale } \\
\text { Avec conjoint, avec enfant(s) } \\
\text { Sans conjoint, avec enfant(s) } \\
\text { Avec conjoint, sans enfant } \\
\text { Sans conjoint, sans enfant }\end{array}$ & $\begin{array}{l}\text { Réf. } \\
\text { n.s. } \\
\text { n.s. } \\
\mathbf{1 , 9 * * *}\end{array}$ & $\begin{array}{l}\text { Réf. } \\
\text { n.s. } \\
\text { n.s. } \\
0,50\end{array}$ & $\begin{array}{l}\text { Réf. } \\
\text { n.s. } \\
\text { n.s. } \\
\text { n.s. }\end{array}$ & $\begin{array}{l}\text { Réf. } \\
\text { n.s. } \\
\text { n.s. } \\
\text { n.s. }\end{array}$ \\
\hline $\begin{array}{l}\text { Caractéristiques de l'aîné } \\
\text { Âge } \\
\text { Situation matrimoniale } \\
\text { Avec conjoint, avec enfant(s) } \\
\text { Sans conjoint, avec enfant(s) } \\
\text { Avec conjoint, sans enfant } \\
\text { Sans conjoint, sans enfant }\end{array}$ & $\begin{array}{l}\text { Réf. } \\
\text { n.s. } \\
-1,11^{\star \star} \\
\text { n.s. }\end{array}$ & $\begin{array}{l}\text { Réf. } \\
\text { n.s. } \\
0,49 \\
\text { n.s. }\end{array}$ & $\begin{array}{l}\text { n.s. } \\
\text { Réf. } \\
\text { n.s. } \\
\text { n.s. } \\
\mathbf{1 , 3 0 * \star \star}\end{array}$ & $\begin{array}{l}\text { n.s. } \\
\text { Réf. } \\
\text { n.s. } \\
\text { n.s. } \\
0,33\end{array}$ \\
\hline $\begin{array}{l}\text { Caractéristiques croisées de l'aîn } \\
\text { Genre } \\
\text { Cadet garçon et aîné garçon } \\
\text { Cadet garçon et aînée fille } \\
\text { Cadet fille et aîné garçon } \\
\text { Cadet fille et aînée fille } \\
\text { Écart d'âge }\end{array}$ & $\begin{array}{l}\text { Réf. } \\
0,54^{*} \\
1,09^{* * *} \\
0,52^{*}\end{array}$ & $\begin{array}{l}\text { Réf. } \\
0,29 \\
0,40 \\
0,28\end{array}$ & $\begin{array}{l}\text { Réf. } \\
\text { n.s. } \\
\text { n.s. } \\
\text { n.s. }\end{array}$ & $\begin{array}{l}\text { Réf. } \\
\text { n.s. } \\
\text { n.s. } \\
\text { n.s. }\end{array}$ \\
\hline $\begin{array}{l}\text { Moins de } 5 \text { ans } \\
\text { Entre } 5 \text { ans et } 10 \text { ans } \\
\text { Plus de } 10 \text { ans }\end{array}$ & 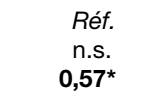 & $\begin{array}{l}\text { Réf. } \\
\text { n.s. } \\
0,35\end{array}$ & $\begin{array}{l}\text { Réf. } \\
\text { n.s. } \\
\text { n.s. }\end{array}$ & $\begin{array}{l}\text { Réf. } \\
\text { n.s. } \\
\text { n.s. }\end{array}$ \\
\hline Activité professionnelle & & & & \\
\hline $\begin{array}{l}\text { Aucun ne travaille à temps plein } \\
\text { L'aîné travaille à temps plein } \\
\text { Le cadet travaille à temps plein } \\
\text { Les deux travaillent à temps plein }\end{array}$ & $\begin{array}{l}\text { Réf. } \\
\text { n.s. } \\
\text { n.s. } \\
\text { n.s. }\end{array}$ & $\begin{array}{l}\text { Réf. } \\
\text { n.s. } \\
\text { n.s. } \\
\text { n.s. }\end{array}$ & $\begin{array}{c}\text { Réf. } \\
\text { n.s. } \\
0,88^{\star *} \\
-\mathbf{1 , 2 1 * *}\end{array}$ & $\begin{array}{l}\text { Réf. } \\
\text { n.s. } \\
0,37 \\
0,47\end{array}$ \\
\hline Niveau d'éducation & & & & \\
\hline $\begin{array}{l}\text { L'un des deux n'a pas fait d'études } \\
\text { supérieures } \\
\text { Les deux ont fait des études } \\
\text { supérieures }\end{array}$ & 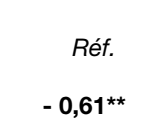 & $\begin{array}{l}\text { Réf. } \\
0,27\end{array}$ & $\begin{array}{r}\text { Réf. } \\
-0,56^{*}\end{array}$ & $\begin{array}{l}\text { Réf. } \\
0,30\end{array}$ \\
\hline
\end{tabular}




\begin{tabular}{|c|c|c|c|c|}
\hline & \multicolumn{2}{|c|}{$\begin{array}{l}\text { Implication du cadet } \\
\text { Équation } n^{\circ} 1\end{array}$} & \multicolumn{2}{|c|}{$\begin{array}{l}\text { Implication de l'aîné } \\
\text { Équation } n^{\circ} 2\end{array}$} \\
\hline & Coefficient & Écart-type & Coefficient & Écart-type \\
\hline $\begin{array}{l}\text { Implication de l'aîné } \\
\text { L'aîné n'est pas impliqué } \\
\text { L'aîné est impliqué }\end{array}$ & $\begin{array}{r}\text { Réf. } \\
-\mathbf{1 , 1 1 ^ { * }}\end{array}$ & $\begin{array}{c}\text { Réf. } \\
0,60\end{array}$ & & \\
\hline Nombre d'observations & 225 & & 225 & \\
\hline \multicolumn{5}{|c|}{$\begin{array}{l}\text { Covariance estimée des perturbations : } \rho=0,76 \\
\text { Écart type estimé de la covariance des perturbations : } \sigma_{\rho}=0,33 \\
\text { Probabilité critique de significativité }: p=0,23\end{array}$} \\
\hline
\end{tabular}

Lecture : le modèle estimé comporte deux équations. La première modélise l'implication du cadet dans la prise en charge du parent

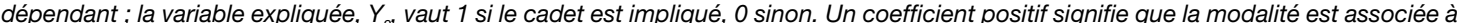
une plus forte probabilité que le cadet s'implique. Ainsi, un cadet sans enfant et sans conjoint (coef $=1,9)$ a une probabilité de s'impliquer plus forte qu'un cadet avec enfants et conjoint (situation de référence). La deuxième équation modélise l'implication de l'aîné ; la variable expliquée, $Y_{1}$, vaut 1 si l'aîné est impliqué. Un coefficient positif signifie que la modalité est associée à une plus forte probabilité que l'aîné s'implique. Ainsi, lorsque le parent souffre d'incapacités sévères (coef =0,54), l'aîné a une probabilité de s'impliquer plus forte que si le parent souffre d'incapacités légères ou modérées (situation de référence).

${ }^{* * *}$ significatif à $1 \%,{ }^{* *}$ significatif à $5 \%,{ }^{*}$ significatif à $10 \%$.

Champ : cadets et aînés appartenant à une fratrie de deux enfants ayant un parent dépendant sans conjoint.

Source : enquête Share, 2004.

Les deux équations sont estimées simultanément en autorisant une corrélation entre les perturbations (supposées normales) des deux régressions. Ceci permet de tenir compte, dans l'estimation, d'une possible hétérogénéité inobservée entre fratries (et non pas seulement entre individus). Ici la covariance des perturbations des deux équations est estimée à 0,76 (les variances étant à priori fixées à 1 par souci d'identifiabilité). II est possible de tester la significativité du coefficient de corrélation entre les perturbations des deux régressions, et donc l'intérêt d'une modélisation sous forme de Probit bivarié. Le test repose sur la comparaison des valeurs prises par la vraisemblance de l'échantillon sous l'hypothèse nulle $(\rho=0)$ et sous l'hypothèse alternative $(\rho \neq 0)$. Ici, la probabilité critique du test est de 0,23 : on a donc 23 chances sur 100 de se tromper si l'on conclut à l'existence d'une hétérogénéité inobservée au niveau des fratries.

Formellement, le modèle s'écrit :

$$
\begin{aligned}
& \left\{\begin{array}{l}
Y_{1 i}^{*}=X_{1 i} \beta_{1}+\varepsilon_{1 i} \\
Y_{2 i}^{*}=X_{2 i} \beta_{2}+\alpha Y_{1 i}+\varepsilon_{2 i}
\end{array}\right. \\
& Y_{1 i}=1 \text { si } Y_{1 i}^{*}>0, \quad 0 \text { sinon } \\
& Y_{2 i}=1 \text { si } Y_{2 i}^{*}>0, \quad 0 \text { sinon }
\end{aligned}
$$

avec

$$
\begin{aligned}
& E\left[\varepsilon_{1 i}\right]=E\left[\varepsilon_{2 i}\right]=0 \\
& \operatorname{Var}\left[\varepsilon_{1 i}\right]=\operatorname{Var}\left[\varepsilon_{2 i}\right]=1 \\
& \operatorname{Cov}\left[\varepsilon_{1 i}, \varepsilon_{2 i}\right]=\rho
\end{aligned}
$$

Dans le modèle retenu ici, l'implication de l'aîné figure comme variable explicative de l'implication du cadet, mais pas l'inverse. Le modèle symétrique a été estimé : d'après les résultats obtenus, l'implication du cadet n'influence pas significativement l'implication de l'aîné. Nous ne présentons pas ici les résultats de l'estimation de ce modèle alternatif qui introduit l'implication du cadet comme variable explicative dans l'équation de l'aîné plutôt que l'inverse. II faut savoir, par ailleurs, qu'il est logiquement impossible d'estimer un modèle qui tienne compte simultanément de l'implication de l'aîné dans l'équation du cadet et de l'implication du cadet dans l'équation de l'aîné (Maddala, 1983).

Pour traiter l'hétérogénéité inobservée au niveau des fratries, il aurait été possible à l'instar de Wolff (2006) d'estimer un modèle à effets fixes mais cela aurait été au prix d'une réduction très sévère du nombre d'observations utilisées puisque ce type de modèle ne s'estime que sur les fratries dont les enfants ont pris des décisions d'implication différentes qui représentent moins d'un tiers de l'échantillon total.

La variable de distance entre la résidence des enfants et celle de leur parent dépendant ne figure pas parmi les variables individuelles insérées dans le modèle. Ce choix a deux motivations. Premièrement, nous traitons la cohabitation comme une modalité d'implication ce qui conduit à une relation déterministe entre la distance et l'implication des enfants pour les situations de cohabitation. Deuxièmement, la distance des enfants à leur parent est probablement largement endogène (Stern, 1995) sans que nous disposions d'instruments idoines pour traiter cette endogénéité. 


\section{Le bilan économique de la France...}

$>$ Et aussi le point sur trois grands thèmes d'actualité :

- Mesure du pouvoir d'achat et sa perception par les ménages,

- Évolution des niveaux de vie, de la productivité et du bien-être en longue période,

- Flux de main-d'oeuvre et les flux d'emplois dans un contexte d'internationalisation.

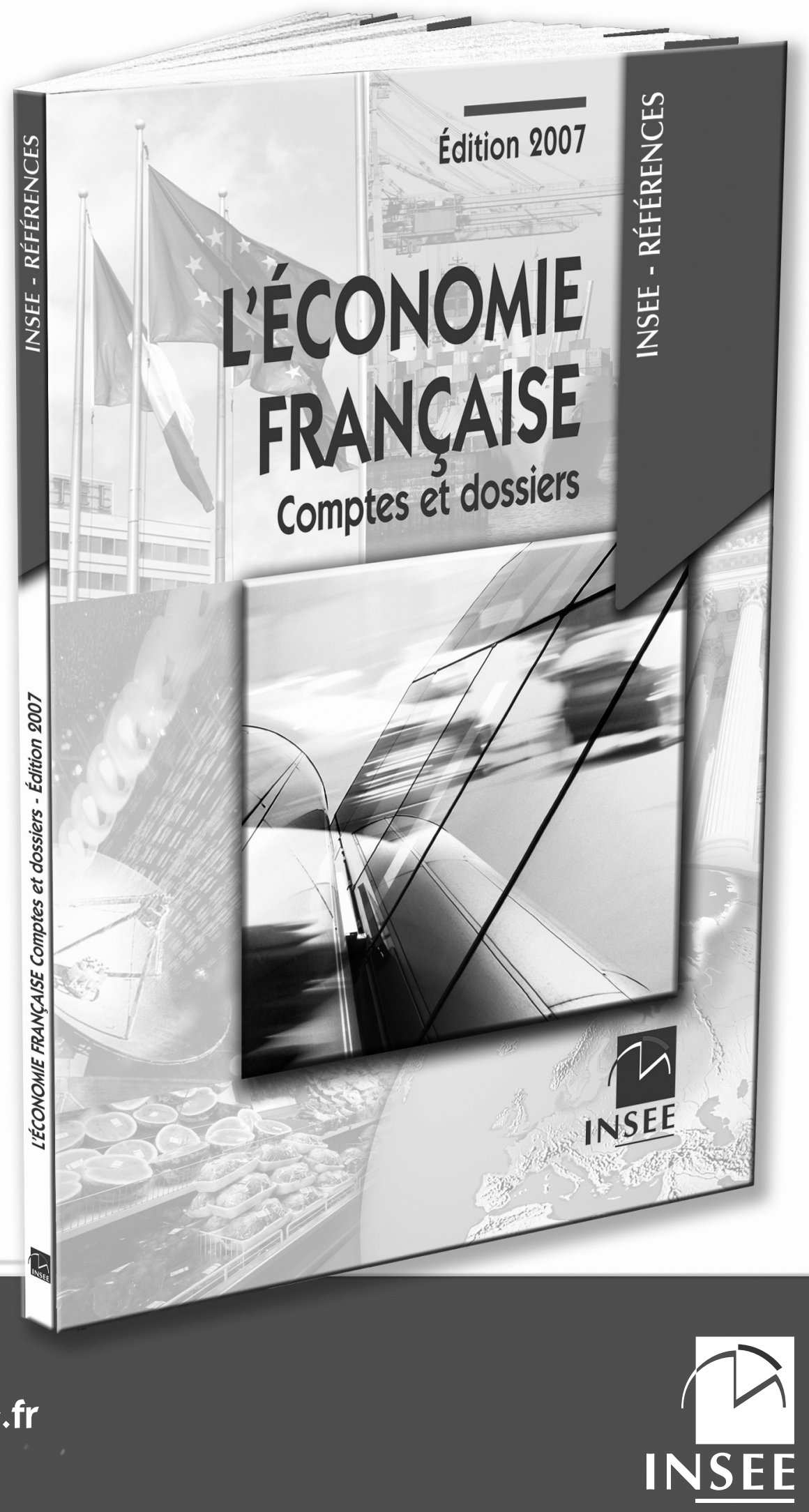

$15 €$ - Collection Insee-Réferences

En vente en librairie,

à l'Insee et sur www.insee.fr

INSEE 